\title{
Nonlinear Dynamic Behaviors of a Marine Rotor-Bearing System Coupled with Air Bag and Floating-Raft
}

\author{
W. Zhao, M. Li, and L. Xiao \\ Department of Mechanics, Xian University of Science and Technology, Shaanxi, Xian 710054, China \\ Correspondence should be addressed to W. Zhao; zhaowenwinner@163.com
}

Received 18 May 2015; Revised 7 July 2015; Accepted 8 July 2015

Academic Editor: Tai Thai

Copyright (c) 2015 W. Zhao et al. This is an open access article distributed under the Creative Commons Attribution License, which permits unrestricted use, distribution, and reproduction in any medium, provided the original work is properly cited.

\begin{abstract}
To understand the nonlinear dynamic mechanism of a rotor-bearing system coupled with air bag and floating-raft, the dynamic characteristics of the system are investigated. This work has two key objectives. First, the vibration mechanism of rotor-bearing system coupled with air bag and floating-raft is investigated by developing a numerical model. Then, the nonlinear dynamics of the system and the effect of several parameters are studied, which includes the steady-state response and its spectrum, the orbit and its Poincaré map, the bifurcation diagram, and largest Lyapunov exponent (LLE). The results show that at low speed the dynamic behavior appears in a single periodic motion, and, with the increase of the speed, the motion becomes quasi-periodic and chaotic. These performances indicate that the air bag and floating-raft introduce some dynamic effects of marine rotor-bearing system.
\end{abstract}

\section{Introduction}

Mechanical vibrations from power machinery are one of the main sources of noises in large vessels and how to reduce them is a matter of great concern. Nowadays, one of the most commonly adopted solutions in power equipment such as marine rotating machinery to suppress the excessive vibrations and reduce its disturbing noise is the use of air bag and floating-raft device.

In recent years, the nonlinear dynamic responses of rotor-bearing system have been analyzed and described in literature [1]. Niu et al. [2] researched the behaviors and the design principles of active floating-raft isolation system and investigated their power transmission [3]. Niu and Song [4] analyzed the influence of the nonlinearity of floating-raft vibration isolation system, and Wang et al. [5] developed the dynamic modeling of elastic floating-raft system on theory of multibody dynamics and structural dynamics. Jiang et al. [6] studied the dynamic effects of elasticity on the raft body and its linear shock response based on the experimental method. Huang [7] studied the dynamic characteristics of marine vibration isolation systems and verified them by carrying out the experimental tests. Stephen [8] discussed the dynamics between a single and a two-stage raft vibration isolation system based on the theory of matrix methodology. Using the finite element method (FEM), Wang et al. [9] analyzed the dynamic characteristics of the floating-raft vibration isolation system of pumping unit. All these studies mainly considered only the linear behavior of the floatingraft and rarely referred to the nonlinear one and do not consider the multimixed air-floating system combined with the rotor-bearing. In addition, the structures of the models cannot be timely changed when the vessel is sailing under the complicated and changeable environment. Therefore, a deeper study of rotor-bearing system coupled with floatingraft isolation device is auspicable [10].

The above-mentioned literature mainly emphasized the linear dynamic behaviors such as the dynamic responses or the design of floating-raft isolation device [11-15]. However, the nonlinear dynamics of the rotor-bearing system coupled with air bag and floating-raft isolation equipment is little mentioned. In previous studies, Zhao and Li [16] developed a mathematic model for a rotor system with vibration isolation equipment based on the theory of short bearing, and Song et al. [17] studied the dynamic reduction modeling and time delay for a vibration isolation system with a flexible base. Therefore, to investigate the effects of nonlinearities on nonperiodic motions of rotor-bearing system, this paper paid 


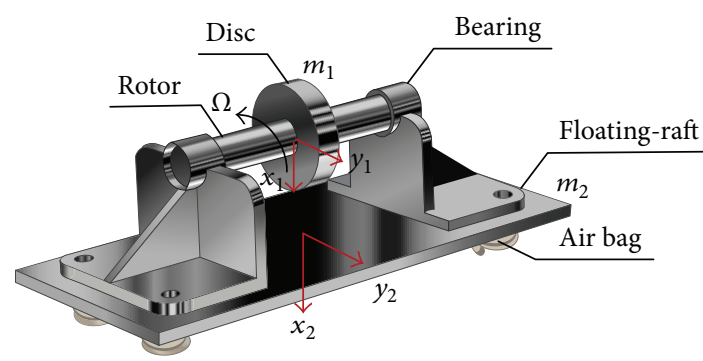

FIGURE 1: Simplified mechanical model.

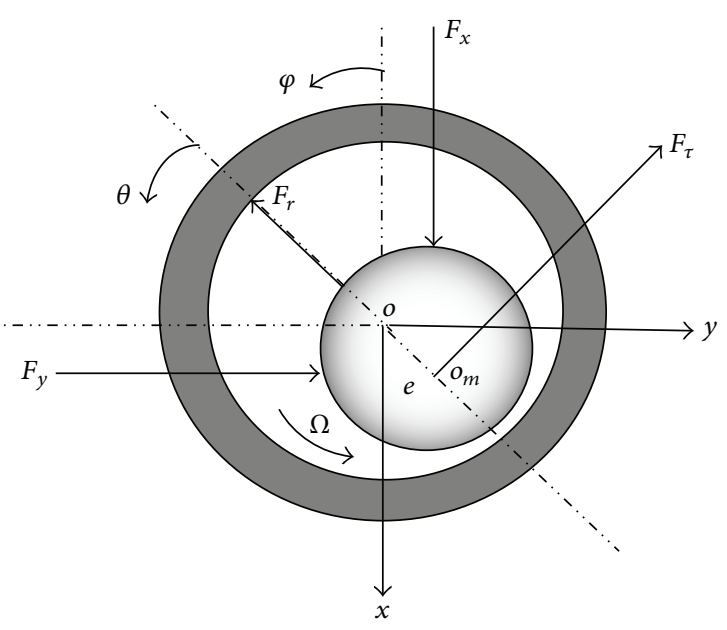

FIGURE 2: Schematic diagram of journal bearing.

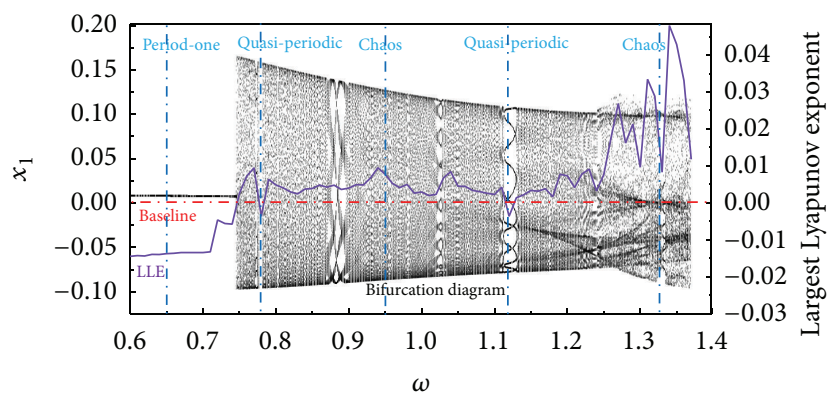

Figure 3: Bifurcation diagram of the steady-state response $x_{1}$ and its largest Lyapunov exponent.

more attention to the dynamics of the rotor system in long journal bearings coupled with air bag and floating-raft device. The nonlinear dynamic equations of the system are solved using computational methods. Steady response, dynamic trajectories, spectrum map, Poincare maps, bifurcation diagrams, and largest Lyapunov exponent are applied to analyze the dynamic behaviors of the system. The nonlinear dynamic mechanical model of the system is built and analyzed in depth to improve its performance of vibration isolation; these studies will lay down the requirements for the engineering design and application of air bag and floating-raft isolation equipment in large vessel.
TABLE 1: The non-dimensional expressions of parameters.

\begin{tabular}{lc}
\hline Parameters & Expression \\
\hline$\alpha$ & $\alpha=\frac{e}{c}$ \\
$n$ & $n=\frac{m_{2}}{m_{1}}$ \\
$\omega$ & $\omega=\Omega \sqrt{\frac{c}{g}}$ \\
$D$ & $D=\frac{d c \Omega}{m_{1} g}$ \\
$\sigma$ & $\sigma=\frac{\bar{\sigma}}{m_{1} \sqrt{g c}}$ \\
$K$ & $K=\frac{k c}{m_{1} g}$ \\
$f_{x}$ & $f_{x} \cos \varphi-f_{t} \sin \varphi$ \\
$\bar{\sigma}$ & $\bar{\sigma}=\frac{6 u B R^{3}}{c^{2}}$ \\
$\bar{x}_{i}$ & $\bar{x}_{i}=\frac{x_{i}}{c}$ \\
$\bar{y}_{i}$ & $\bar{y}_{i}=\frac{y_{i}}{c}$ \\
$f_{j}$ & $f_{j}=\frac{F_{j}}{m_{1} g}$ \\
$f_{y}$ & $\lambda=\frac{B}{2 R}$ \\
& $-f_{r} \sin \varphi+f_{t} \cos \varphi$ \\
\hline
\end{tabular}

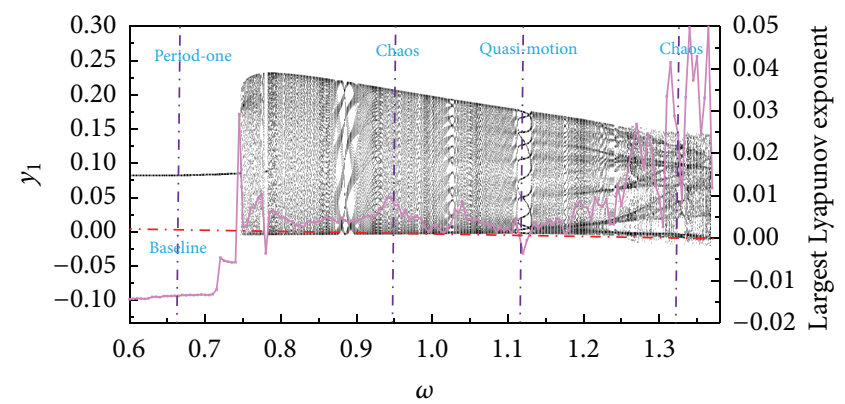

FIGURE 4: Bifurcation diagram of the steady-state response $y_{1}$ and its largest Lyapunov exponent.

\section{Mathematical Model}

Figure 1 shows the rotor-bearing system coupled with multimixed air bag and floating-raft isolation equipment. Floatingraft and rotor are considered as a rigid body, while the air bag is regarded as a spring with vertical and horizontal deformations.

2.1. Motion Equations. According to Figure 1, based on Newton's law, the motion equations of the rotor-bearing system 


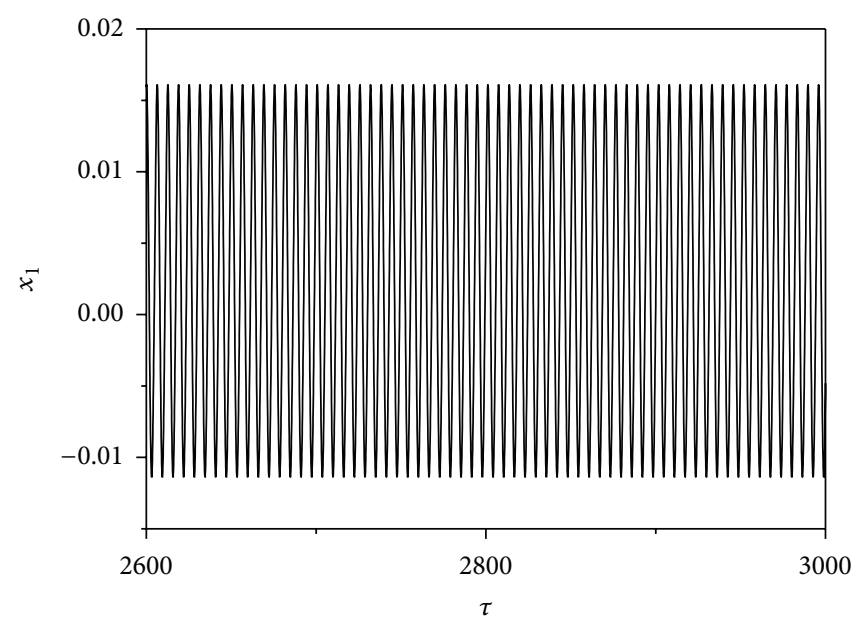

(a)

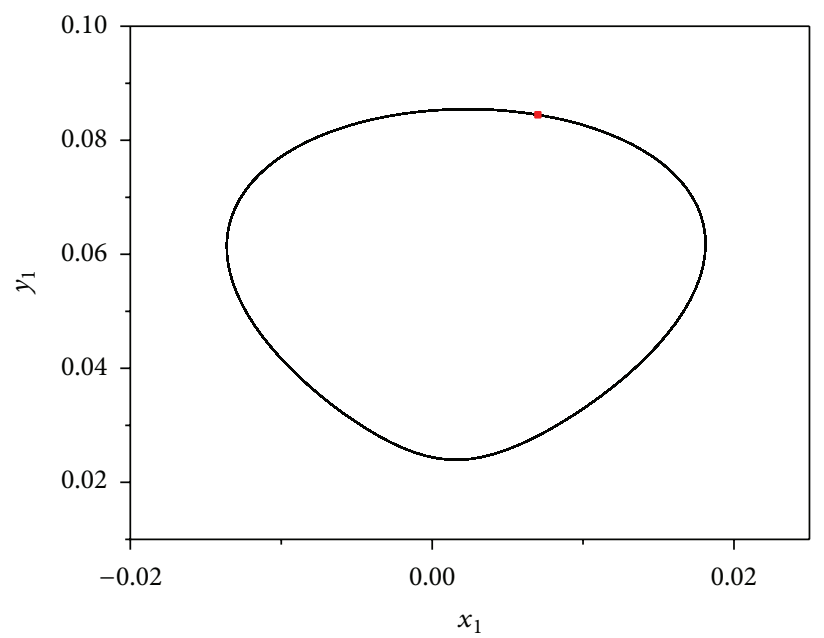

(c)

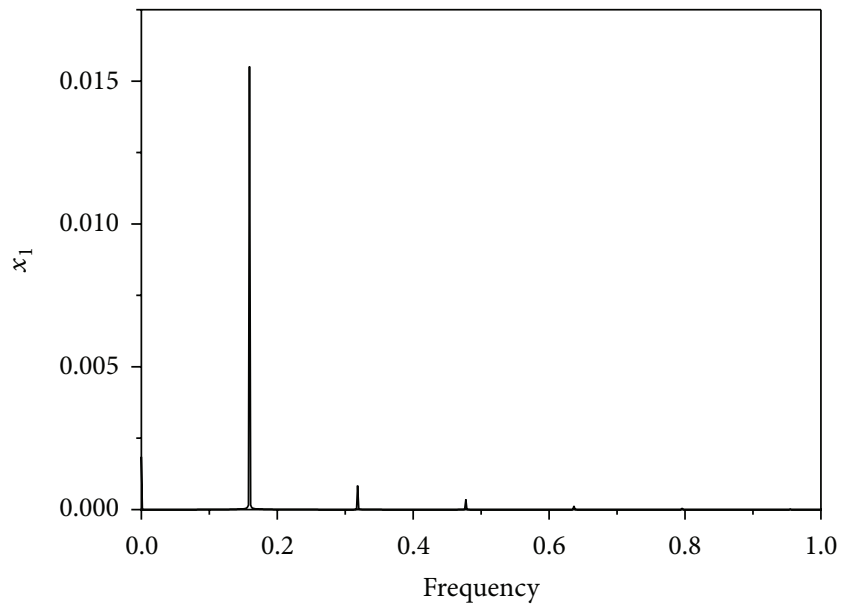

(b)

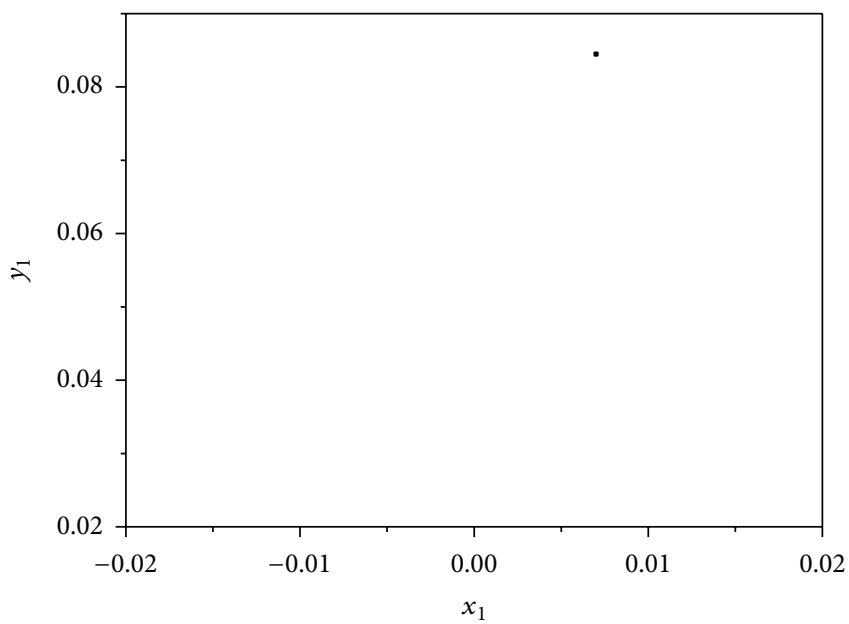

(d)

Figure 5: See $\omega=0.65$. (a) Displacement response. (b) Frequency spectrum. (c) Rotor orbit. (d) Poincaré maps.

coupled with the air bag and floating-raft device can be expressed as

$$
\begin{aligned}
m_{1} \ddot{x}_{1}( & t) \\
= & m_{1} g \\
& \quad+F_{x}\left[\left(x_{1}-x_{2}\right),\left(y_{1}-y_{2}\right),\left(\dot{x}_{1}-\dot{x}_{2}\right),\left(\dot{y}_{1}-\dot{y}_{2}\right)\right] \\
& \quad+m_{1} e \Omega^{2} \cos \Omega t, \\
m_{1} \ddot{y}_{1}(t) & \\
= & F_{y}\left[\left(x_{1}-x_{2}\right),\left(y_{1}-y_{2}\right),\left(\dot{x}_{1}-\dot{x}_{2}\right),\left(\dot{y}_{1}-\dot{y}_{2}\right)\right] \\
& \quad+m_{1} e \Omega^{2} \sin \Omega t, \\
m_{2} \ddot{x}_{2} & (t) \\
= & m_{2} g \\
\quad & F_{x}\left[\left(x_{1}-x_{2}\right),\left(y_{1}-y_{2}\right),\left(\dot{x}_{1}-\dot{x}_{2}\right),\left(\dot{y}_{1}-\dot{y}_{2}\right)\right]
\end{aligned}
$$

$$
-k_{21} x_{2}(t)-d_{21} \dot{x}_{2}(t),
$$

$$
\begin{aligned}
m_{2} \ddot{y}_{2}(t) & \\
= & -F_{y}\left[\left(x_{1}-x_{2}\right),\left(y_{1}-y_{2}\right),\left(\dot{x}_{1}-\dot{x}_{2}\right),\left(\dot{y}_{1}-\dot{y}_{2}\right)\right] \\
& -k_{22} y_{2}(t)-d_{22} \dot{y}_{2}(t),
\end{aligned}
$$

where $m_{1}, m_{2}$ are the mass of disc, the mass of floating-raft, $e$ is eccentricity, $k$ is the stiffness of air bag, $d$ is the damping, $\Omega$ is the rotor speed, and $F x$ and $F y$ are the nonlinear oil forces in $x$ and $y$ directions, respectively. The mathematical model of the 4-DOFs system is shown in Figure 1, and the coordinate system and notation are shown in Figure 2.

2.2. Oil Film Forces. Figure 2 illustrates the schematic diagram of journal bearing, in which $F_{r}$ and $F_{\tau}$ are the nonlinear oil forces in radial and tangential directions, respectively. This paper considers the hydrodynamic long journal bearing 


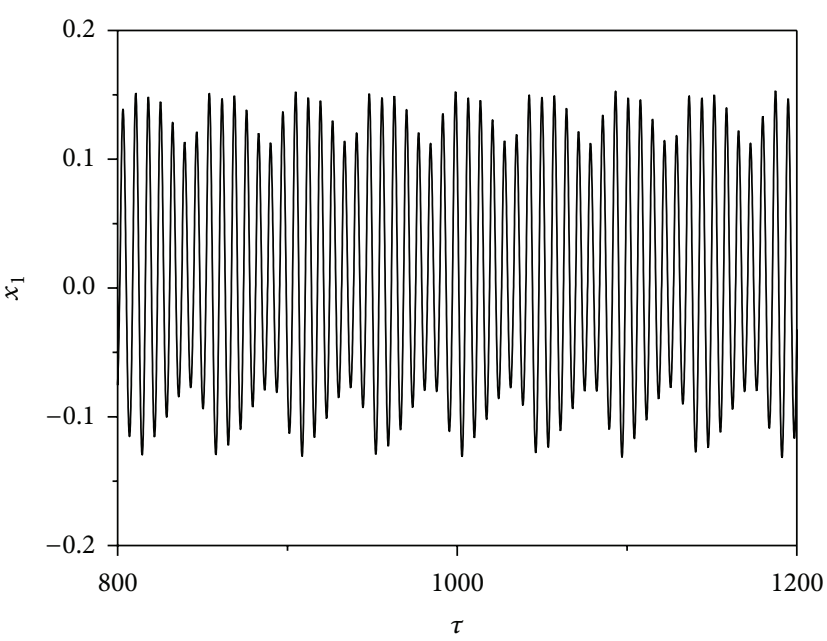

(a)

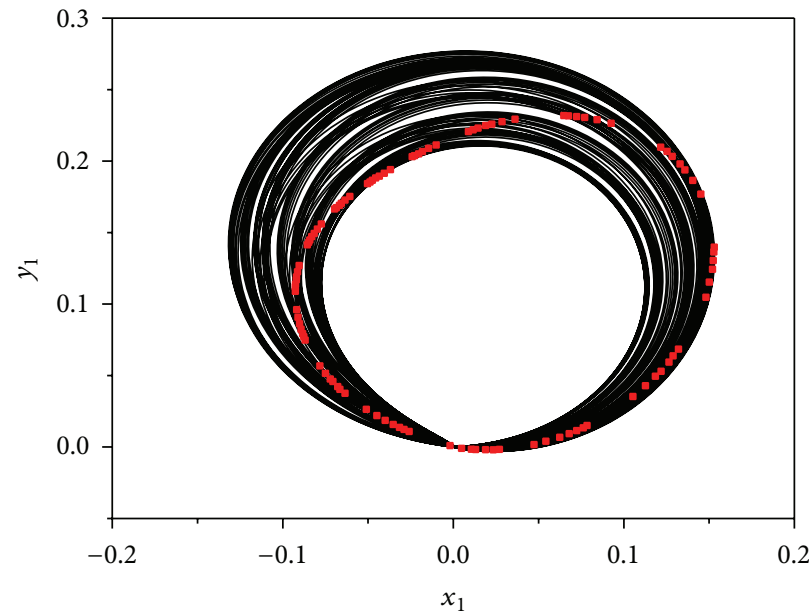

(c)

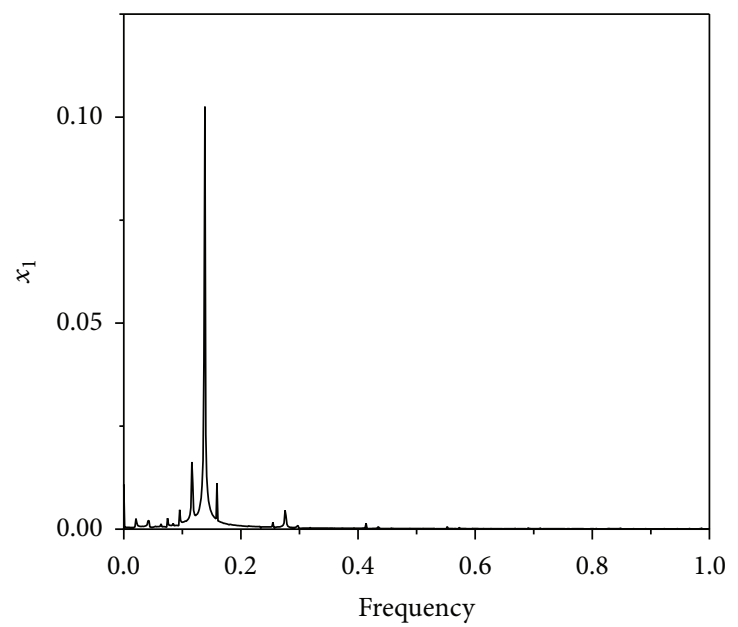

(b)

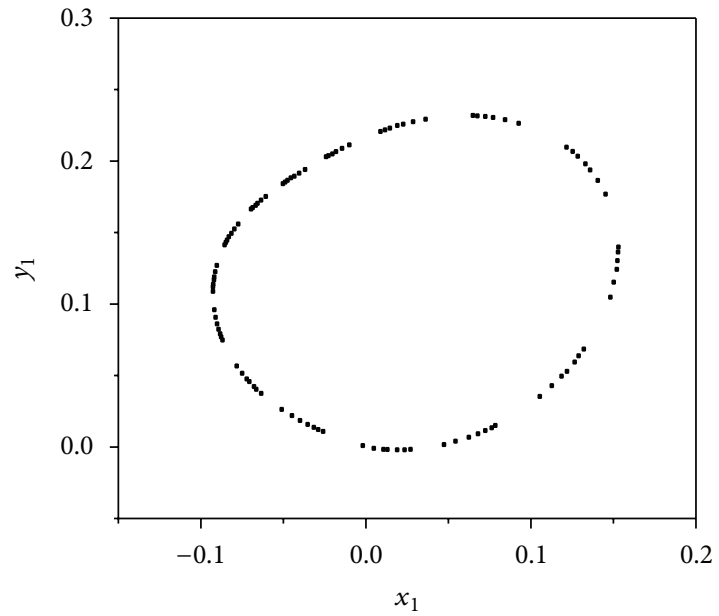

(d)

Figure 6: See $\omega=0.78$. (a) Displacement response; (b) frequency spectrum; (c) rotor orbit; (d) Poincaré maps.

theory; thus, the Reynolds equation for the oil film pressure $p$ is

$$
\frac{1}{R_{b}^{2}} \frac{\partial}{\partial \varphi}\left(\frac{h^{3}}{u} \frac{\partial p}{\partial \varphi}\right)=6(\Omega-2 \dot{\theta}) \frac{\partial h}{\partial \varphi}+12 \dot{e} \cos \varphi
$$

where $h=c+e \cos \theta=c(1+\varepsilon \cos \theta)$.

Under the semi-Sommerfeld condition $\theta=0, p=0$, and $\theta=\pi, p=0$, the oil film forces in radial and tangent directions are obtained

$$
\begin{aligned}
& F_{r}=6 u B R\left(\frac{R}{c}\right)^{2}\left[\left(\Omega-2 \frac{d \theta}{d t}\right) E_{1}+2 \frac{d \varepsilon}{d t} E_{2}\right], \\
& F_{\tau}=6 u B R\left(\frac{R}{c}\right)^{2}\left[\left(\Omega-2 \frac{d \theta}{d t}\right) E_{3}+2 \frac{d \varepsilon}{d t} E_{4}\right],
\end{aligned}
$$

where

$$
\begin{aligned}
& E_{1}=\frac{2 \alpha^{2}}{\left(1-\alpha^{2}\right)\left(2+\alpha^{2}\right)}, \\
& E_{2}=\frac{1}{\left(1-\alpha^{2}\right)^{3 / 2}\left[\frac{\pi}{2}-\frac{8}{\pi\left(2+\alpha^{2}\right)}\right],} \\
& E_{3}=\frac{\pi \alpha}{\left(1-\alpha^{2}\right)^{1 / 2}\left(2+\alpha^{2}\right)}, \\
& E_{4}=\frac{2 \alpha}{\left(1-\alpha^{2}\right)\left(2+\alpha^{2}\right)} .
\end{aligned}
$$

The oil film forces in (3) can be expressed as

$$
\begin{gathered}
F_{x}\left(x_{1}-x_{2}, y_{1}-y_{2}, \dot{x}_{1}-\dot{x}_{2}, \dot{y}_{1}-\dot{y}_{2}\right) \\
\quad=-F_{r} \cos \varphi-F_{t} \sin \varphi
\end{gathered}
$$




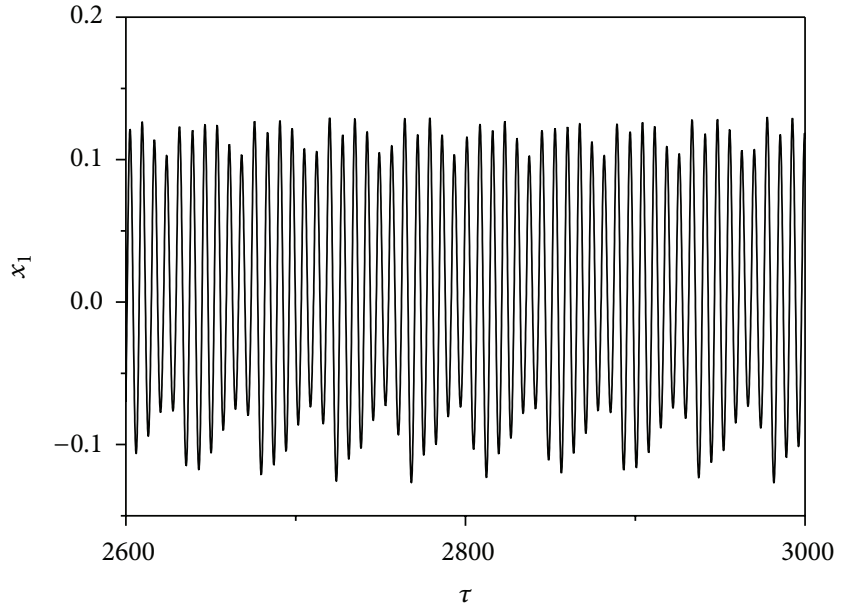

(a)

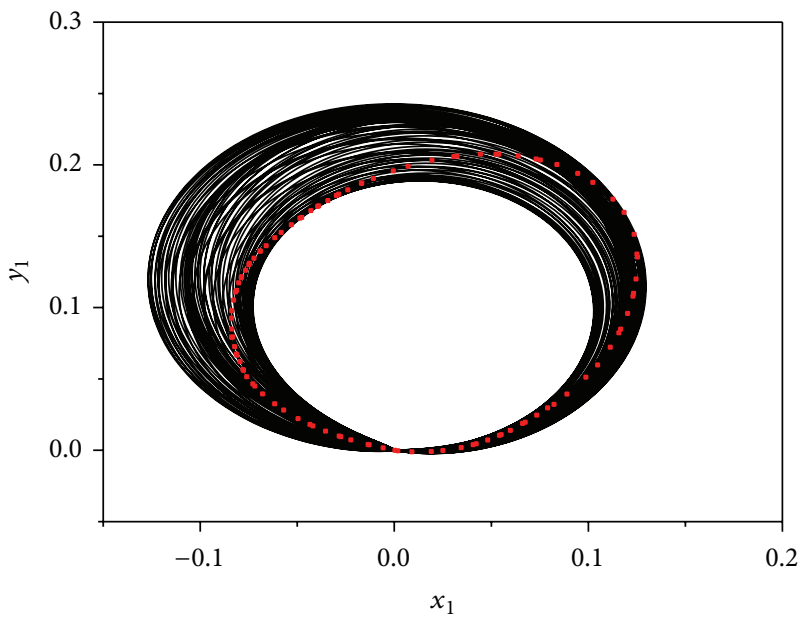

(c)

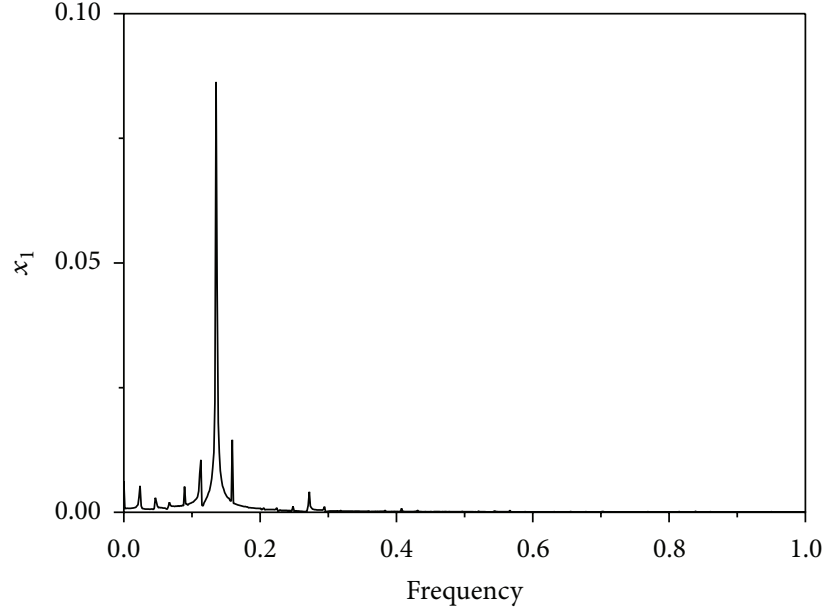

(b)

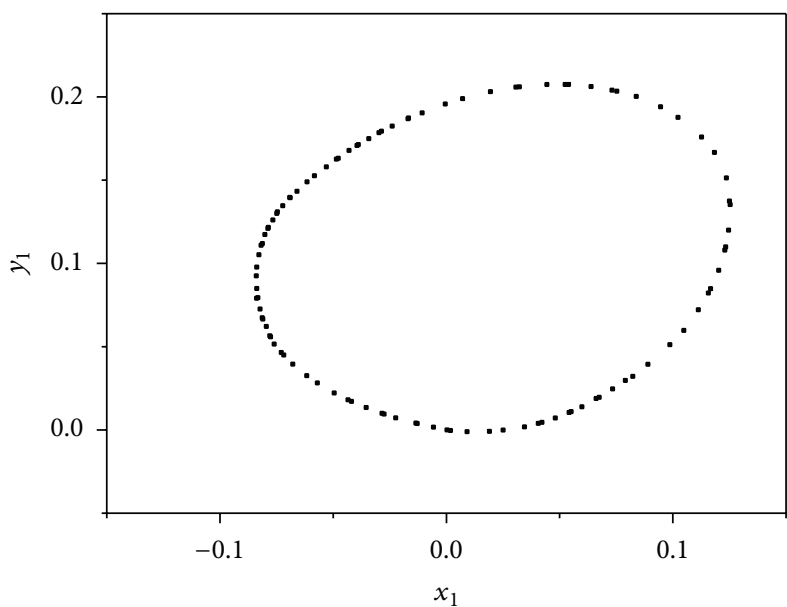

(d)

Figure 7: See $\omega=0.95$. (a) Displacement response. (b) Frequency spectrum. (c) Rotor orbit. (d) Poincaré maps.

$$
\begin{aligned}
& F_{y}\left(x_{1}-x_{2}, y_{1}-y_{2}, \dot{x}_{1}-\dot{x}_{2}, \dot{y}_{1}-\dot{y}_{2}\right) \\
& =-F_{r} \sin \varphi+F_{t} \cos \varphi,
\end{aligned}
$$

where $F_{x}$ and $F_{y}$ are the nonlinear oil film forces components of journal bearing in $x$ and $y$ direction, respectively.

2.3. Nondimensional Motion Equation. To simplify, the nondimensional notations are introduced and the bearing clearance, weight of disc, and time are considered. Thus, the nondimensional expressions of parameters are shown in Table 1. According to $d x / d t=\dot{x}, d x / d \tau=x^{\prime}, \ldots$. Equation (3) can be rewritten into a more convenient nondimensional form:

$$
\begin{aligned}
& f_{r}=\sigma \omega\left[\left(1-2 \varphi^{\prime}\right) E_{1}+2 e^{\prime} E_{2}\right], \\
& f_{\tau}=\sigma \omega\left[\left(1-2 \varphi^{\prime}\right) E_{3}+2 e^{\prime} E_{4}\right] .
\end{aligned}
$$

Thereby, the nondimensional formulation of motion can be written as

$$
\begin{aligned}
& \bar{x}_{1}^{\prime \prime}(\tau)=\frac{1}{\omega^{2}}+\frac{f_{x}}{\omega^{2}}+\alpha \cos \tau, \\
& \bar{y}_{1}^{\prime \prime}(\tau)=\frac{f_{y}}{\omega^{2}}+\alpha \sin \tau, \\
& \bar{x}_{2}^{\prime \prime}(\tau)=\frac{1}{\omega^{2}}-\frac{f_{x}}{n \omega^{2}}-\frac{K \bar{x}_{2}(\tau)}{n \omega^{2}}-\frac{D \bar{x}_{2}^{\prime}(\tau)}{n \omega^{2}}, \\
& \bar{y}_{2}^{\prime \prime}(\tau)=-\frac{f_{y}}{n \omega^{2}}-\frac{K \bar{y}(\tau)}{n \omega^{2}}-\frac{D \bar{y}_{2}^{\prime}(\tau)}{n \omega^{2}} .
\end{aligned}
$$

Equation (7) can be discussed on the state space and the four second-order equations can be easily converted into eight first-order ones

$$
\begin{aligned}
& \dot{Z}_{1}(\tau)=Z_{2}(\tau)=\dot{\bar{x}}_{1}(\tau) \\
& \dot{Z}_{2}(\tau)=\ddot{\bar{x}}_{1}(\tau)=\frac{1}{\omega^{2}}+\frac{f_{x}}{\omega^{2}}+\alpha \cos \tau, \\
& \dot{Z}_{3}(\tau)=Z_{4}(\tau)=\dot{\bar{y}}_{1}(\tau)
\end{aligned}
$$




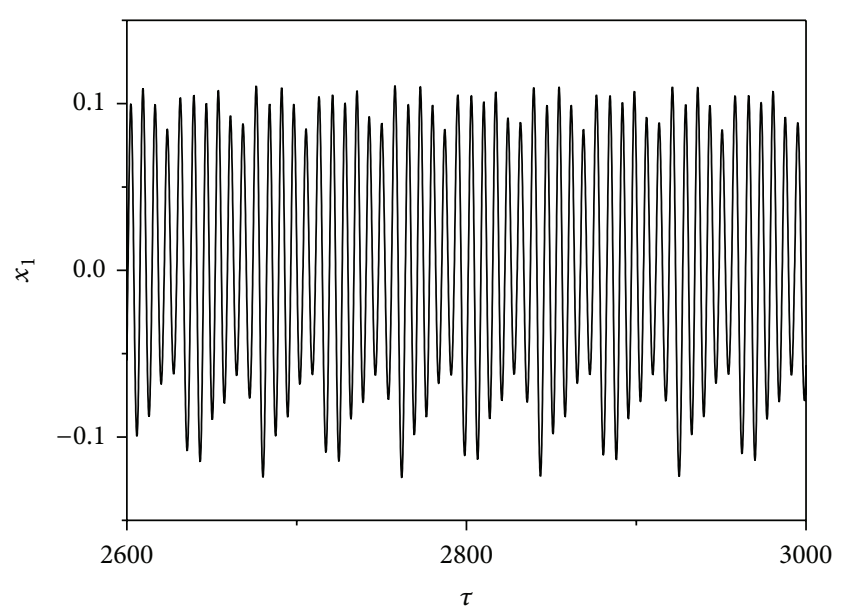

(a)

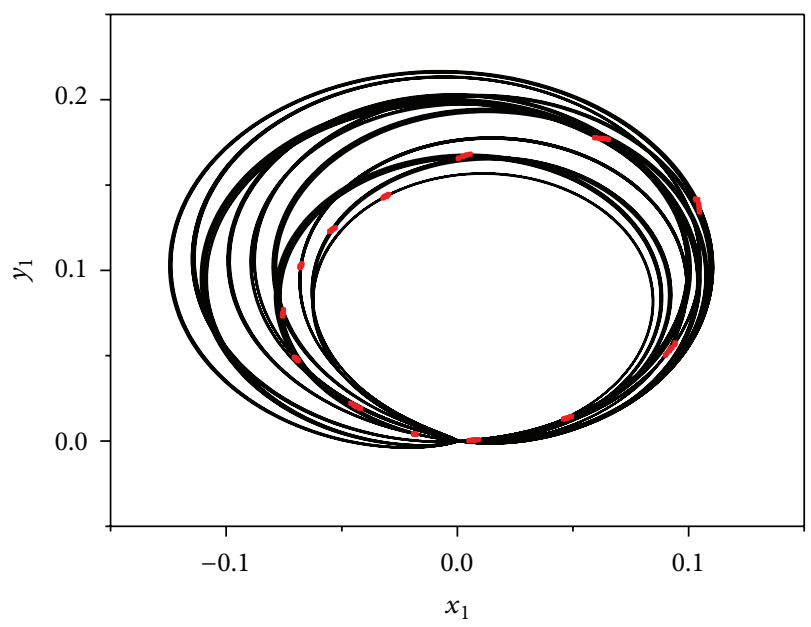

(c)

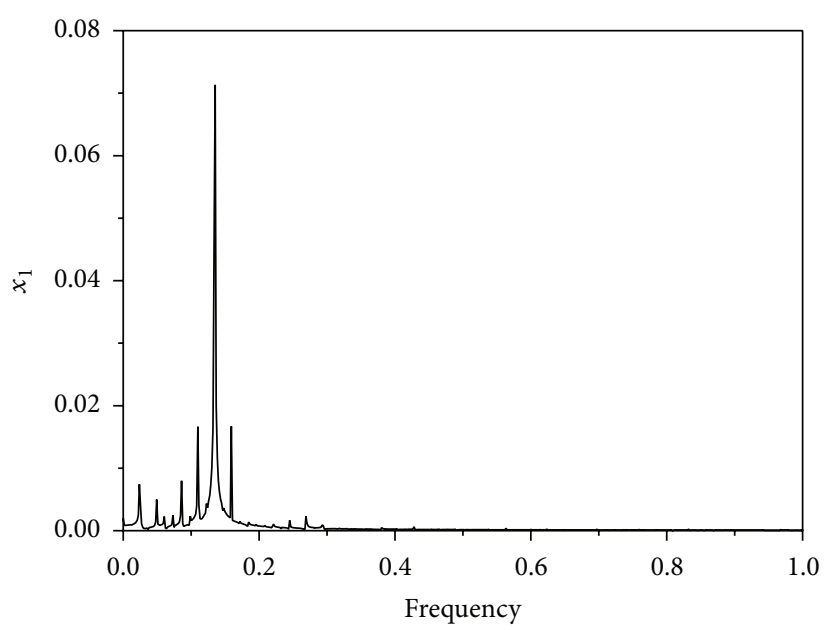

(b)

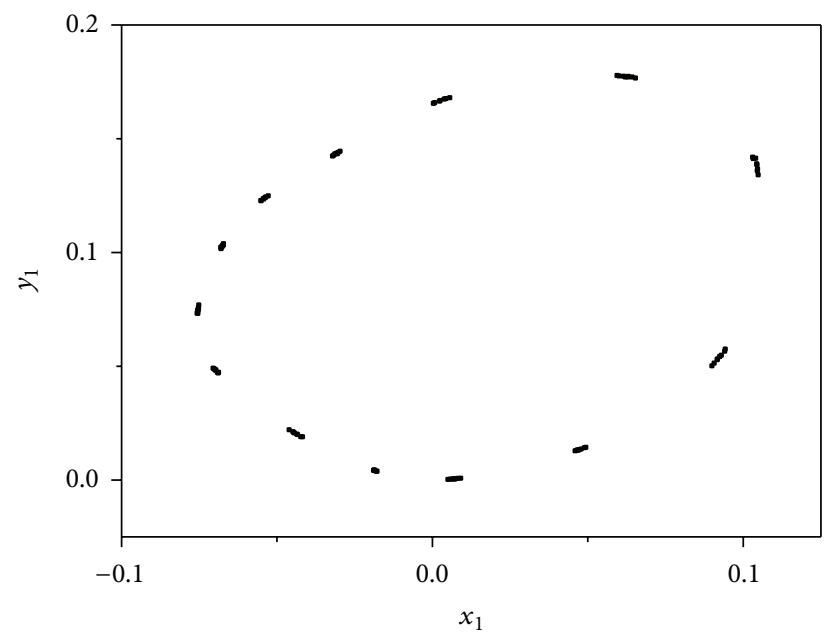

(d)

Figure 8: See $\omega=1.12$. (a) Displacement response. (b) Frequency spectrum. (c) Rotor orbit. (d) Poincaré maps.

$$
\begin{aligned}
\dot{Z}_{4}(\tau) & =\ddot{\bar{y}}_{1}(\tau)=\frac{f_{y}}{\omega^{2}}+\alpha \sin \tau \\
\dot{Z}_{5}(\tau) & =Z_{6}(\tau)=\dot{\bar{x}}_{2}(\tau) \\
\dot{Z}_{6}(\tau) & =\ddot{\bar{x}}_{2}(\tau) \\
& =\frac{1}{\omega^{2}}-\frac{f_{x}}{n \omega^{2}}-\frac{K}{n \omega^{2}} Z_{5}(\tau)-\frac{D}{n \omega^{2}} Z_{6}(\tau), \\
\dot{Z}_{7}(\tau) & =Z_{8}(\tau)=\dot{\bar{y}}_{2}(\tau), \\
\dot{Z}_{8}(\tau) & =\ddot{\bar{y}}_{2}(\tau)=-\frac{f_{y}}{n \omega^{2}}-\frac{K}{n \omega^{2}} Z_{7}(\tau)-\frac{D}{n \omega^{2}} Z_{8}(\tau),
\end{aligned}
$$

where $\alpha, \omega, n, K$, and $D$ are the nondimensional eccentricity, rotor speed, mass ratio, stiffness, and damping, respectively; $\sigma$ is the Sommerfeld number; $f_{x}$ and $f_{y}$ are the nondimensional nonlinear oil film forces. Consequently, the dynamic characteristics of the system depend on the nondimensional parameters $n, \alpha, \sigma, \omega, K$, and $D$.

\section{Nonlinear Dynamic Analysis}

Equation (8) is strongly nonlinear and no analytical method is applicable. Thus, to solve (8) the present work adopts, the Runge-Kutta integration routine with variable step. The parameters of the system are $n=10, \alpha=0.1, \sigma=3, K=12$, and $D=0.1$, and the following initial conditions are set as $x_{1}=0.2, \dot{x}_{1}=0.0, y_{1}=0.1, \dot{y}_{1}=0.0, x_{2}=0.1, \dot{x}_{2}=0.0$, $y_{2}=0.1$, and $\dot{y}_{2}=0.0$.

3.1. Numerical Results. Equation (8) describes a nonlinear nonautonomous system with multidimensional rotorbearing system, the nonlinear oil film, and nonlinear functions in terms of $\sin \varphi$ and $\cos \varphi$. This section introduces the numerical solution using the Runge-Kutta method.

Figure 3 shows the bifurcation diagram of rotor-bearing system coupled with the air bag and the floating-raft isolation 


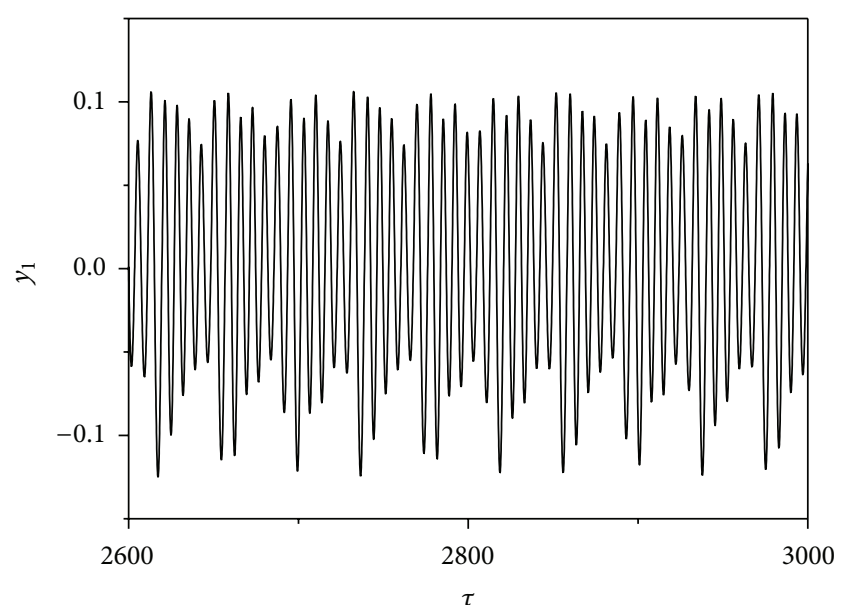

(a)

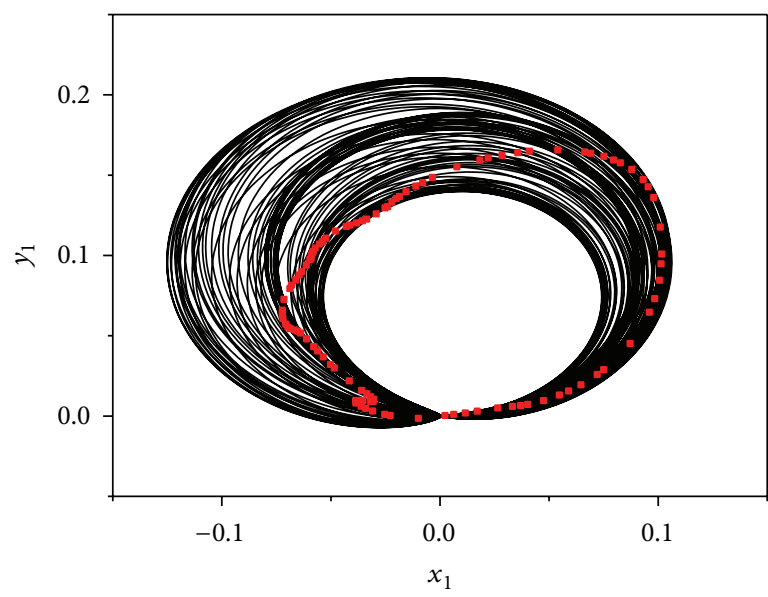

(c)

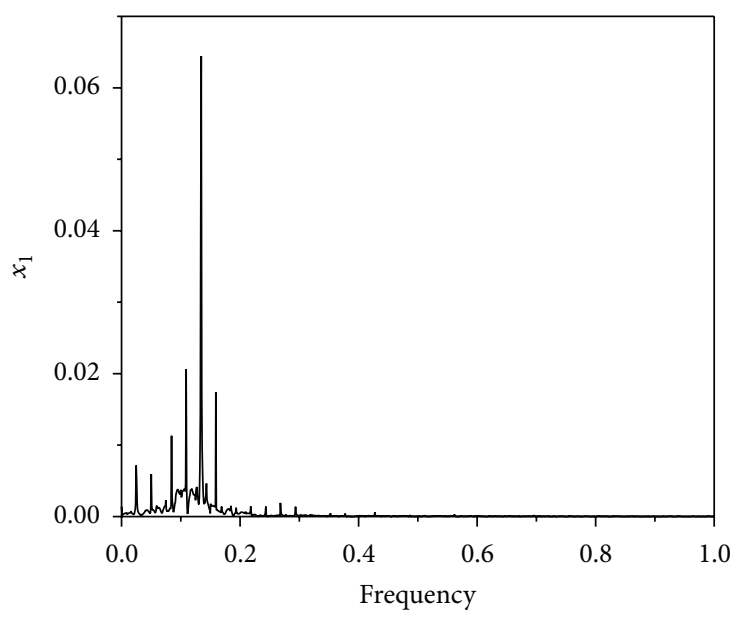

(b)

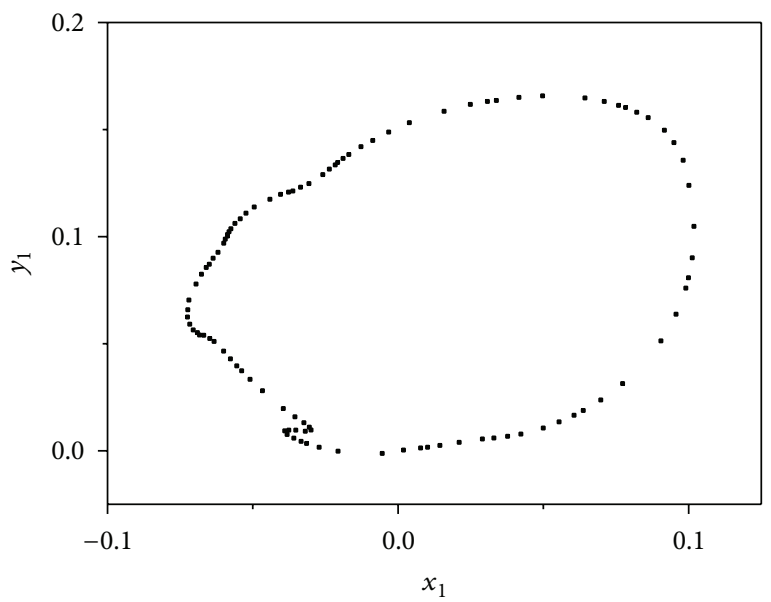

(d)

Figure 9: See $\omega=1.20$. (a) Displacement response. (b) Frequency spectrum. (c) Rotor orbit. (d) Poincaré maps.

equipment. The diagram refers to the displacement response $x_{1}$ when motion parameter $\omega$ varies from 0.600 to 1.370 . The same figure also plots the largest Lyapunov exponent of the bifurcation diagram. The results indicate that the steady-state responses at low frequencies are synchronous with the rotor speed. When $\omega$ lies within the range $0.600-0.741$, the diagram shows single periodic motions similar to dynamic behavior in the linear system. However, the bifurcation of quasi-periodic motion occurs at $\omega$ around $0.780-0.800$ and 1.110-1.130; in these cases, the dynamic characteristics are mainly expressed by the quasi-periodic oscillation. With the rapid increments of rotor speed, the chaotic motion will rise; for rotation speed greater than 1.378, the chaotic motion clearly emerges, and at the same time the jump phenomenon of the amplitudes occurs and the rotor directly impacts the bearing bush. This bifurcation point and the type of bifurcation are the same as the above result obtained by numerical integration.

Figure 4 shows the bifurcation diagram of the same system for the displacement response $y_{1}$ with respect to the motion parameter $\omega$, in which $\omega$ varies from 0.600 to 1.370 . The same figure plots LLE of the bifurcation diagram. With the increase of the rotor speed, the dynamic behaviors change from single-one motion to quasi-motion to chaotic motion. The largest Lyapunov exponents converge to positive values, confirming the rising chaos.

Figure 5 illustrates the dynamic response and its frequency spectrum and rotor orbit and its Poincaré map at about $\omega=0.650$, which shows one point in the Poincaré map that the motion is in the state of a period-one motion at this rotor speed. Its largest Lyapunov exponent is -0.013731 . Thus, we are sure that it is period-one motion at $\omega=$ 0.650 from the simulation results of steady-state response, frequency spectrum, rotor orbit, Poincaré maps, and the largest Lyapunov exponent.

Figure 6 shows the oscillations at $\omega=0.780$, in which the nonlinear dynamic behaviors are the quasi-periodic motion and the displacement response (Figure 6(a)) highlights the beat vibration. The frequency spectrum shows a harmonic components; the steady-state response of this system is confined to an annular region and the rotor makes complex movements as shown in (c). Points on the Poincaré maps are an approximate closed curve, the dynamic characteristic of 


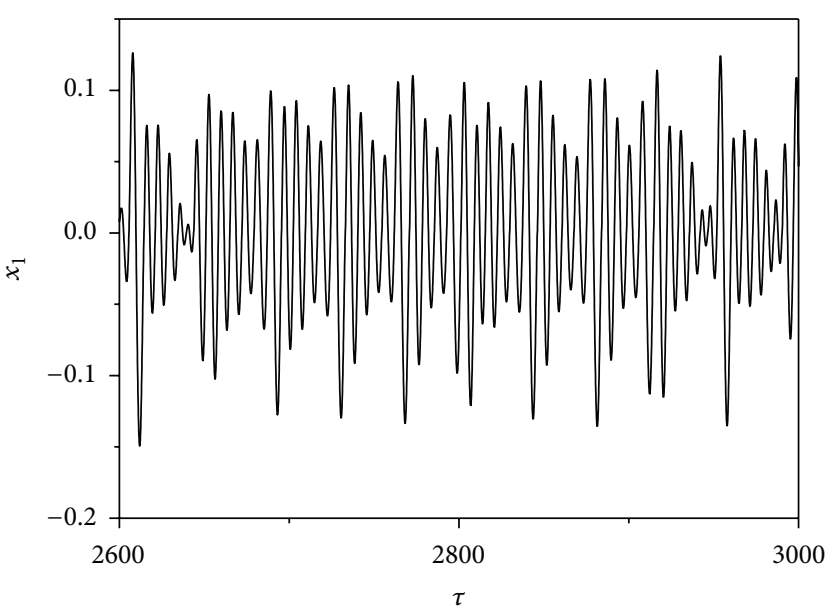

(a)

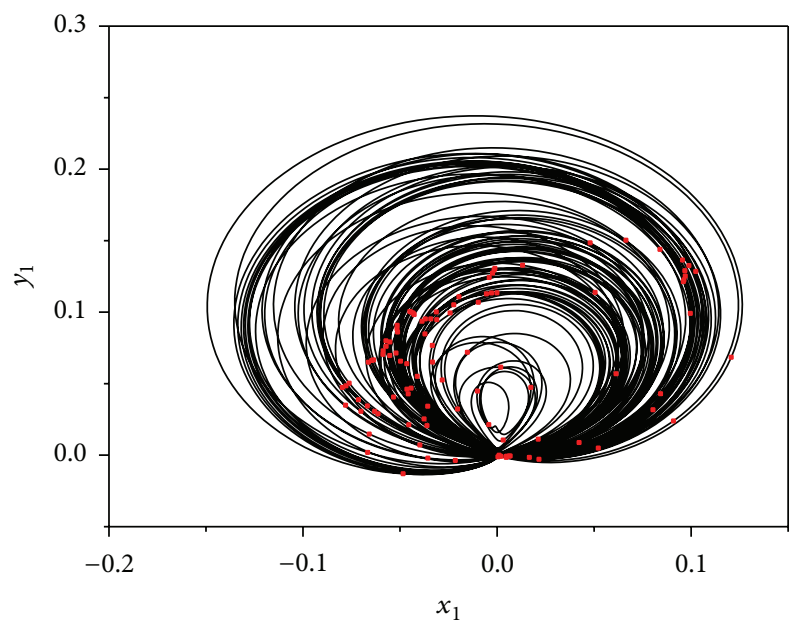

(c)

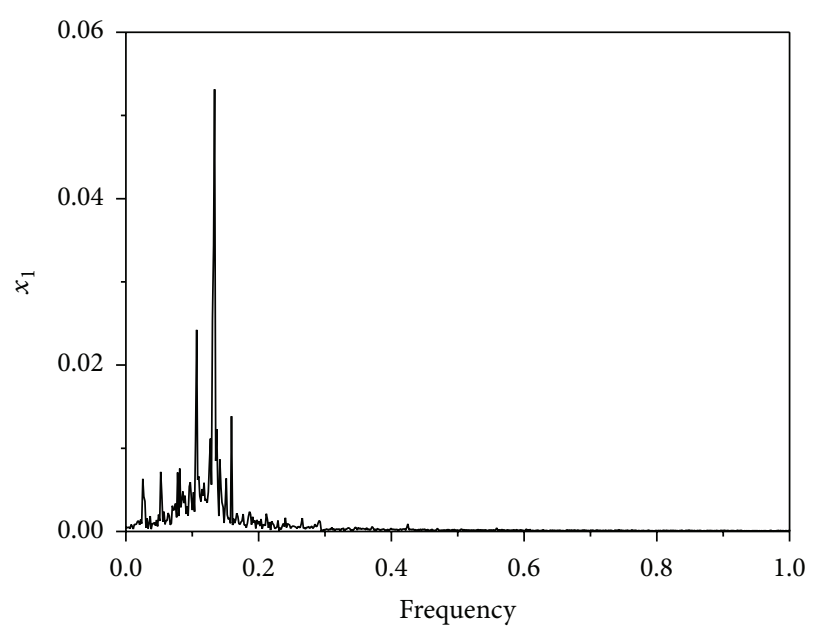

(b)

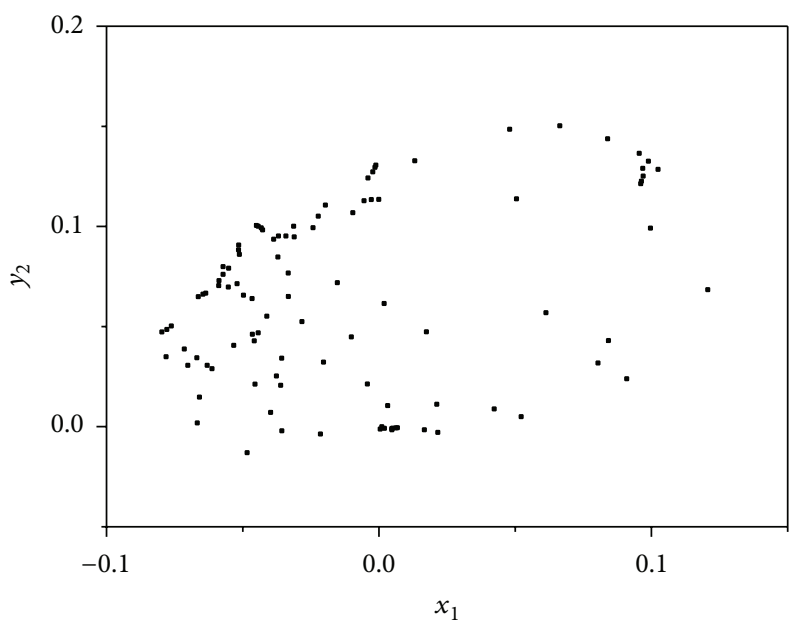

(d)

Figure 10: See $\omega=1.30$. (a) Displacement response. (b) Frequency spectrum. (c) Rotor orbit. (d) Poincaré maps.

the quasi-periodic motion at this rotor speed. Its largest Lyapunov exponent is negative and equals -0.00361 . Therefore, simulation results identify the quasi-periodic motion of the system at $\omega=0.780$.

Figure 7 indicates the results of the simulations at $\omega=$ 0.950. The analysis of the Poincaré map highlights that at this rotor speed the motion is chaotic. In fact, from the orbit graph, it can be seen that the steady-state response of this system is confined to an annular region and can make irregular movements, while largest Lyapunov exponent LLE = 0.007765 .

Figure 8 shows the dynamic behaviors of the system at $\omega=1.120$. The orbit graph shows how this dynamic response confined to an annular region and the rotor makes complex movements. The points on the Poincaré map present a discrete and unclose curve, while its largest Lyapunov exponent is 0.00361 , confirming that at $\omega=1.120$ the motion is chaotic.

Figure 9 analyzes the oscillations at $\omega=1.200$, in which the quasi-periodic motion is prominent. There exists some continuous spectrum in the frequency spectrum structure and rotor orbit trajectory is messy. Furthermore, the Poincaré diagram shows many irregular points, and its largest Lyapunov exponent diagram reflects the positive value showing that at $\omega=1.200$ the motion is chaotic.

Figure 10 illustrates the steady response and its frequency spectrum and rotor obit and Poincaré map when rotor speed is $\omega=1.300$. The frequency spectrum shows a series of continuous spectra, the orbit of rotor is more regular than the one at lower speed, and Poincaré diagram shows irregular points. In addition, its largest Lyapunov exponent is 0.009671. Therefore, one can conclude that when $\omega=1.300$ the system has a nonlinear dynamic behavior and shows chaotic motion.

3.2. Parametric Analysis. As a structural parameter, the effect of the floating-raft mass is very important to be considered in the design.

Figure 11 illustrates the bifurcation diagram of floatingraft forced by an unbalanced excitation for the displacement responses $x_{2}$ and $y_{2}$ with respect to the motion parameter $\omega$; rotor speed $\omega$ varies from 0.200 to 1.300 in certain parameters 


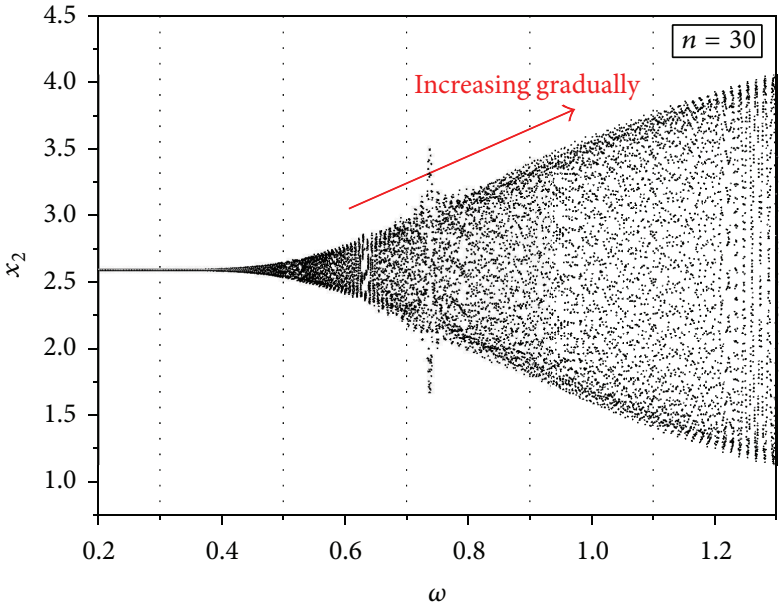

(a)

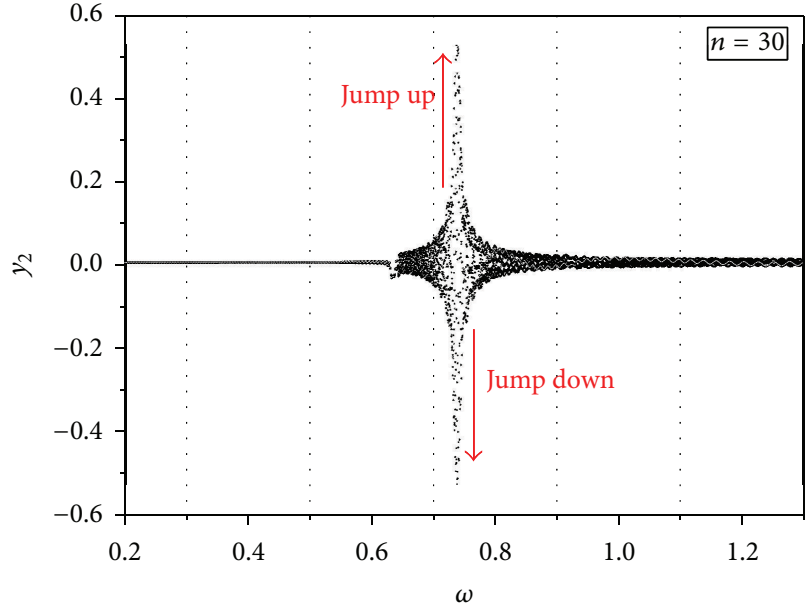

(b)

FIGURE 11: Steady-state response of displacement when $n=30$ : (a) $x_{2}$ and (b) $y_{2}$.

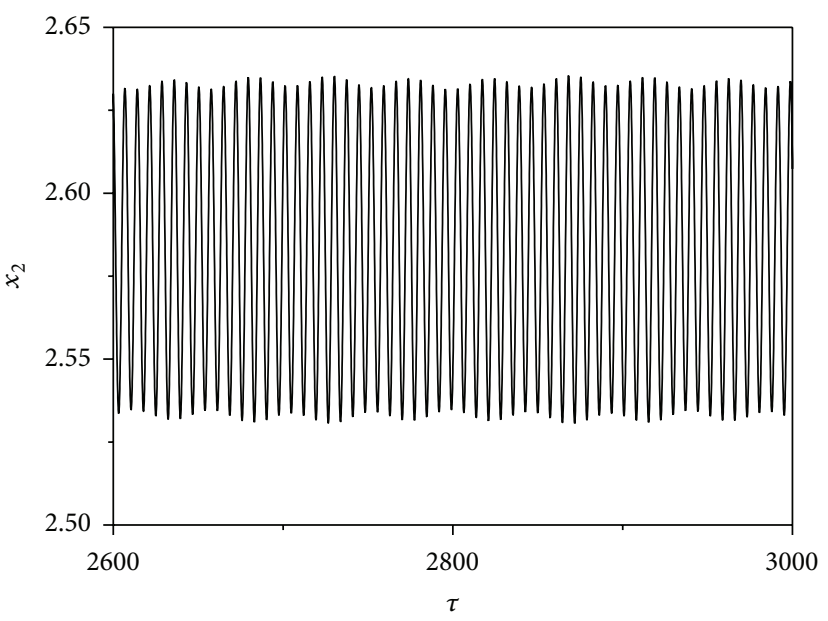

(a)

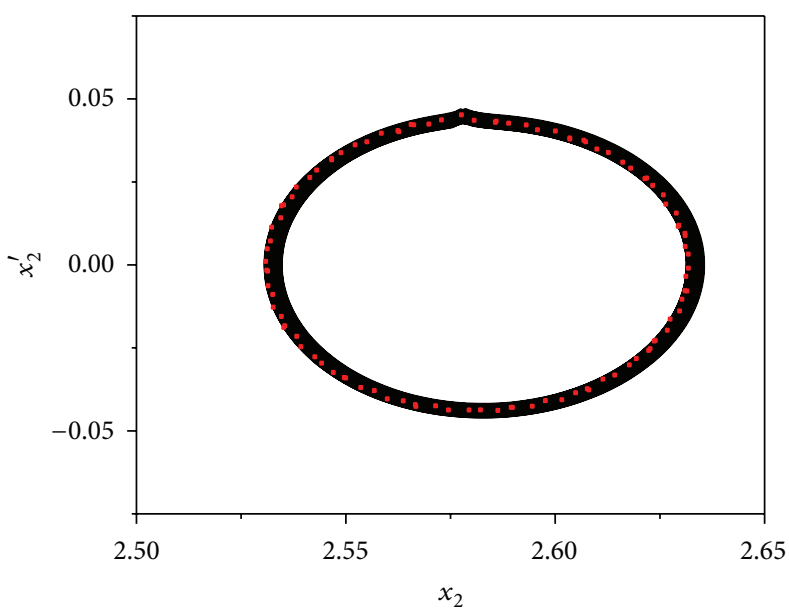

(c)

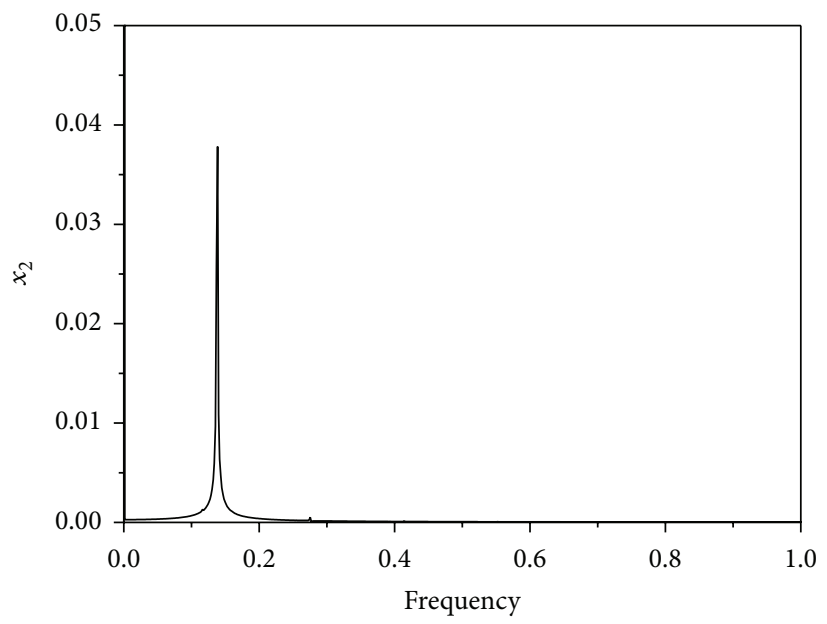

(b)

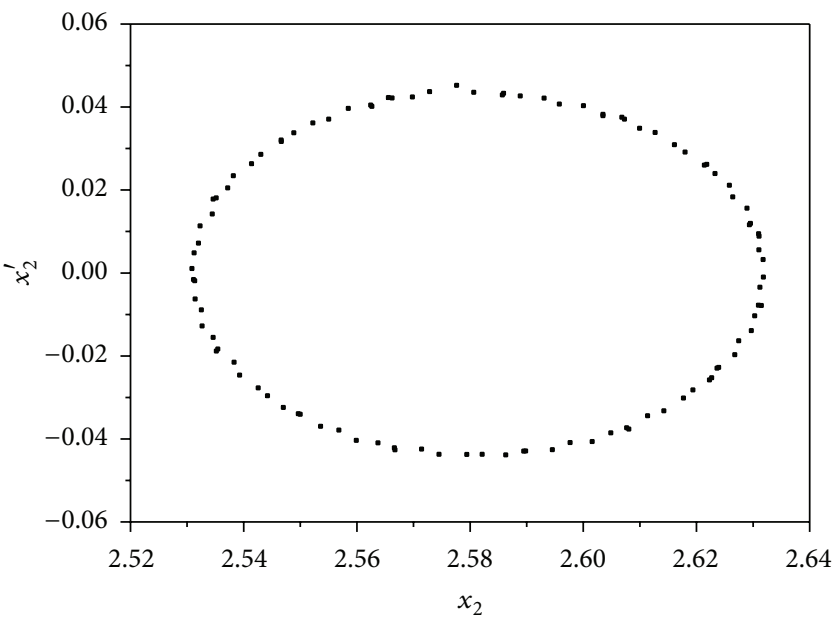

(d)

Figure 12: See $\omega=0.70$. (a) Displacement response. (b) Frequency spectrum. (c) Phase-portrait plots. (d) Poincaré maps. 


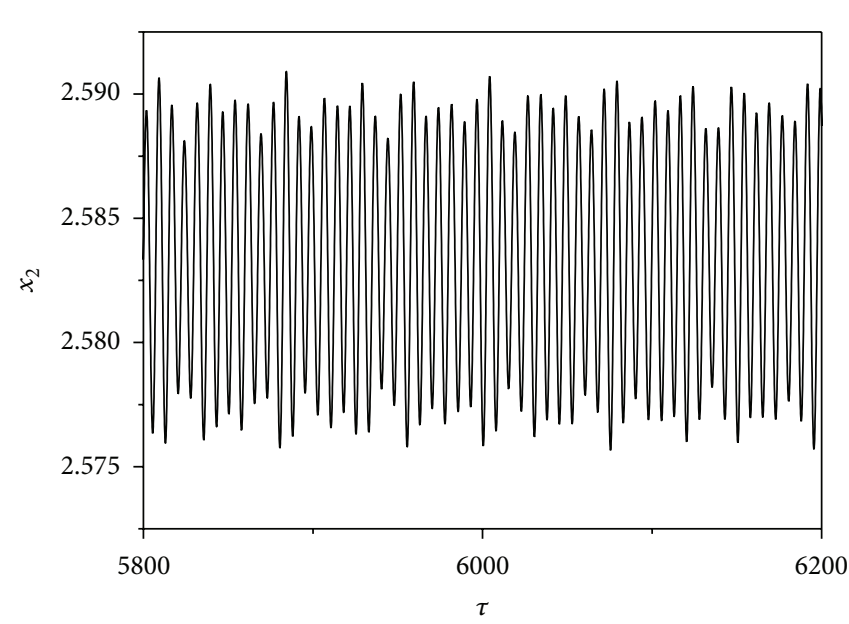

(a)

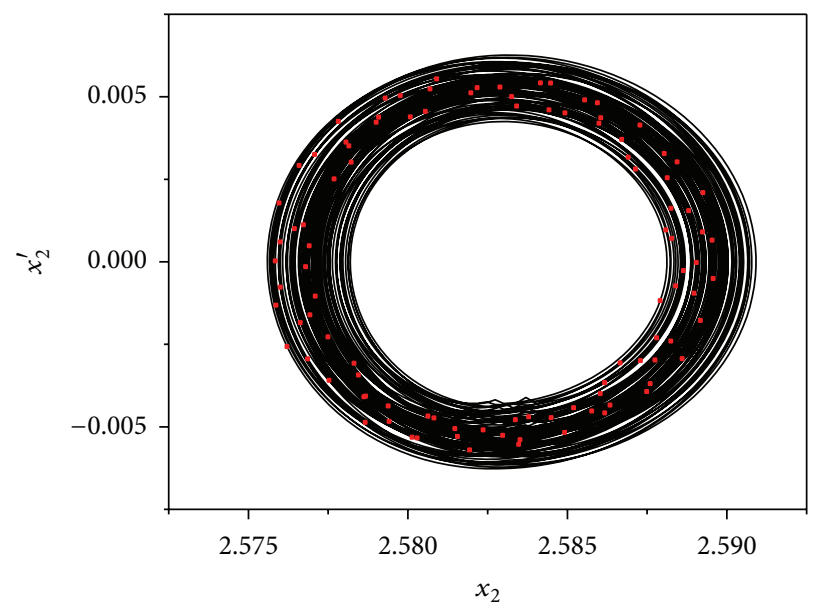

(c)

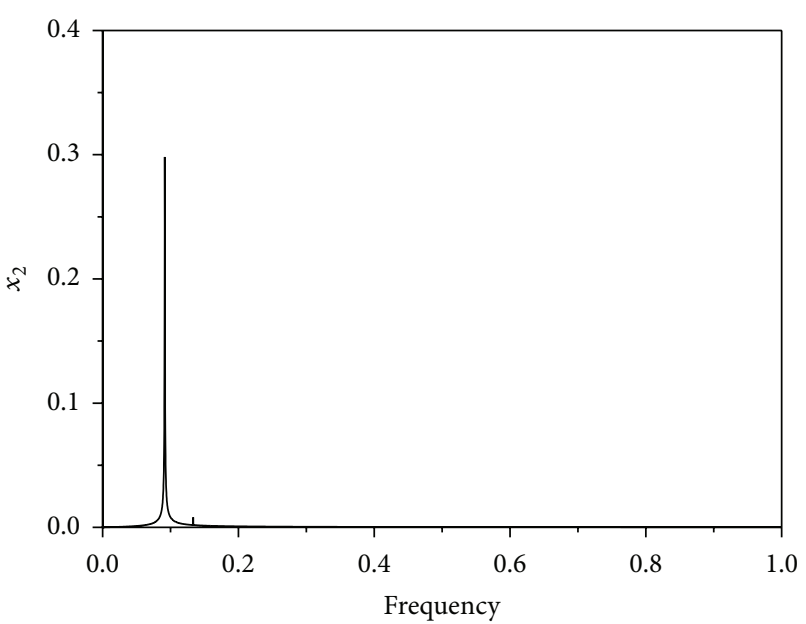

(b)

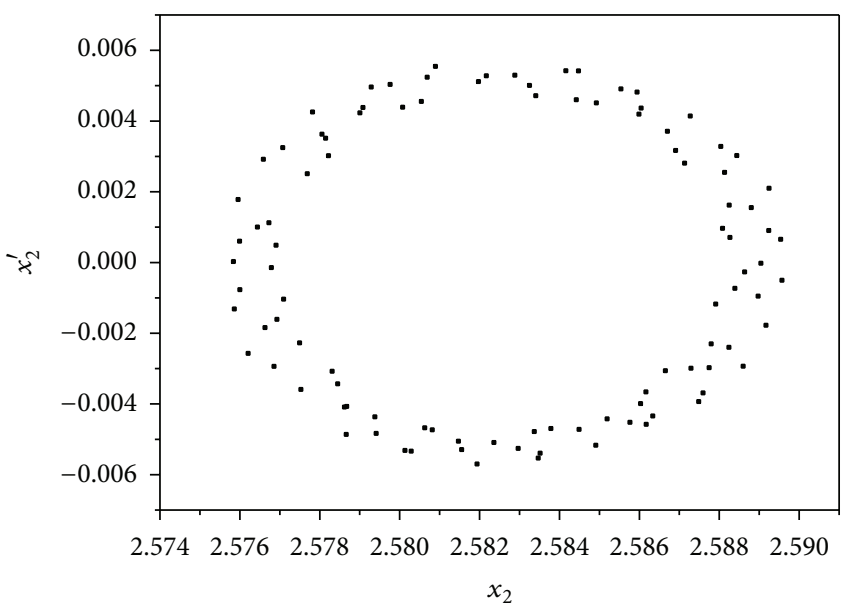

(d)

Figure 13: See $\omega=1.10$. (a) Displacement response. (b) Frequency spectrum. (c) Phase-portrait plots. (d) Poincaré maps.

for $n=30$. With the increase of the rotor speed, the analysis of bifurcation diagram shows that dynamic behaviors in $x_{2}$ and $y_{2}$ directions can be considered as single-one motion, quasimotion, and chaotic motion. With the increase of rotor speed, the amplitude of air-floating increases gradually, and there is a sudden increment of both $x_{2}$ and $y_{2}$ at $\omega=0.737$.

Figure 12 illustrates the dynamic response and its frequency spectrum, phase-portrait plots and Poincaré map at $\omega=0.70$. The points on the Poincaré maps are a close curve, the steady-state response of this system is confined to an annular region and the phase-portrait plot highlights the irregular movements of the rotor. Thus, the motion at this rotor speed is chaotic.

Figure 13 indicates the dynamic response and its frequency spectrum, phase-portrait plots, and Poincaré map at $\omega=1.10$. The steady-state response of this system is confined to an annular region and makes a complex movement from the phase diagram; the points on Poincaré maps are very messy. Thus, the motion states a chaotic motion at this rotor speed.
Figure 14 analyzes the system at $\omega=1.30$. The steady-state response of this system is confined to an annular region and movements are complex from the orbit graph, while points on Poincaré maps are very sophisticated. Thus, at this rotor speed the state is chaotic.

Figure 15 illustrates the bifurcation diagram of floatingraft forced by an unbalanced excitation for the displacement responses $x_{2}$ and $y_{2}$ with respect to the motion parameter $\omega$. Rotor speed varies within the range $0.20-1.30$ in certain parameters for $n=50$. The analysis of the bifurcation diagram shows that with the increase of the rotor speed the dynamic behaviors in $x_{2}$ and $y_{2}$ directions switch from single-one motion to quasi-motion to chaotic motion.

Figure 16 clearly shows that at $\omega=0.80$ the nonlinear dynamic behaviors are the chaotic motion. Phase-portrait plots are messy. Besides, many irregular points on the Poincaré maps are quite intricate, and therefore the motion at this rotating speed ratio $\omega=0.80$ is chaotic.

Figure 17 illustrates the dynamic response and its frequency spectrum, phase-portrait plots, and Poincaré map at 


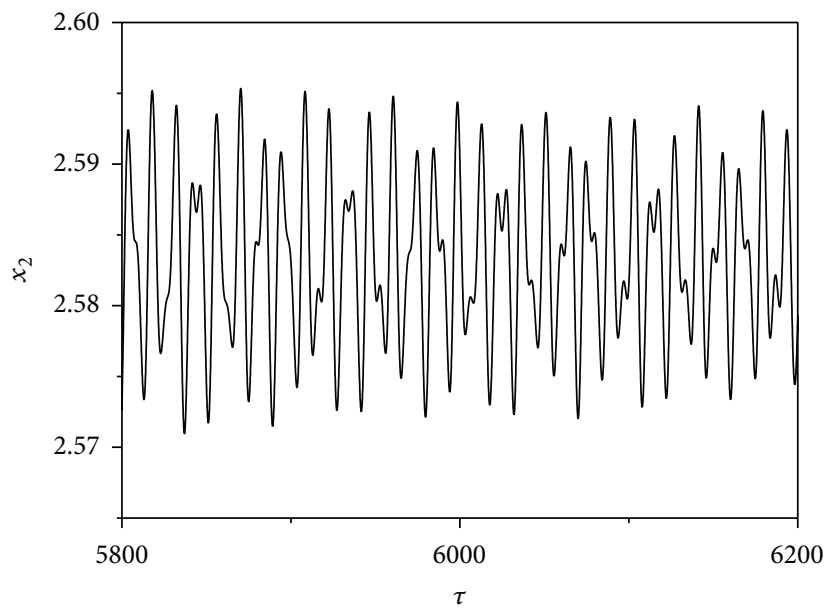

(a)

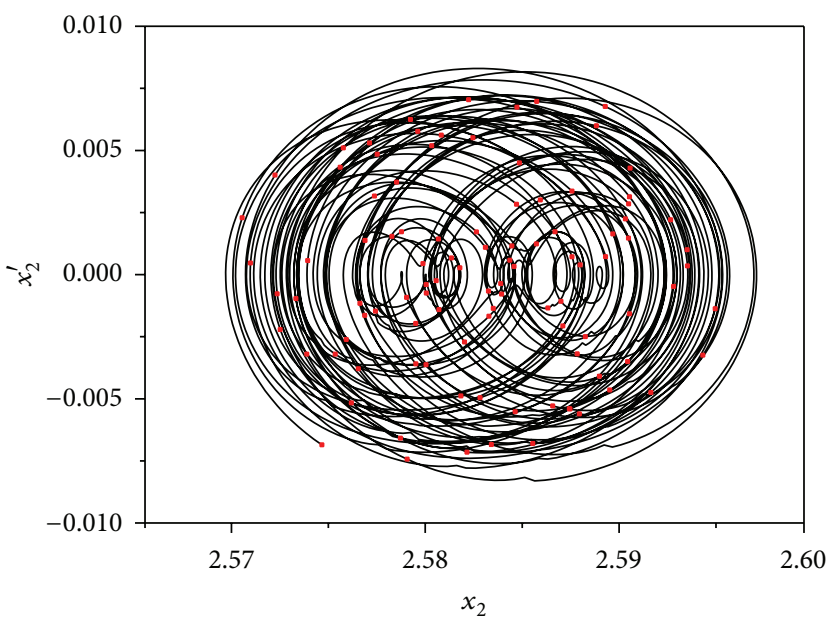

(c)

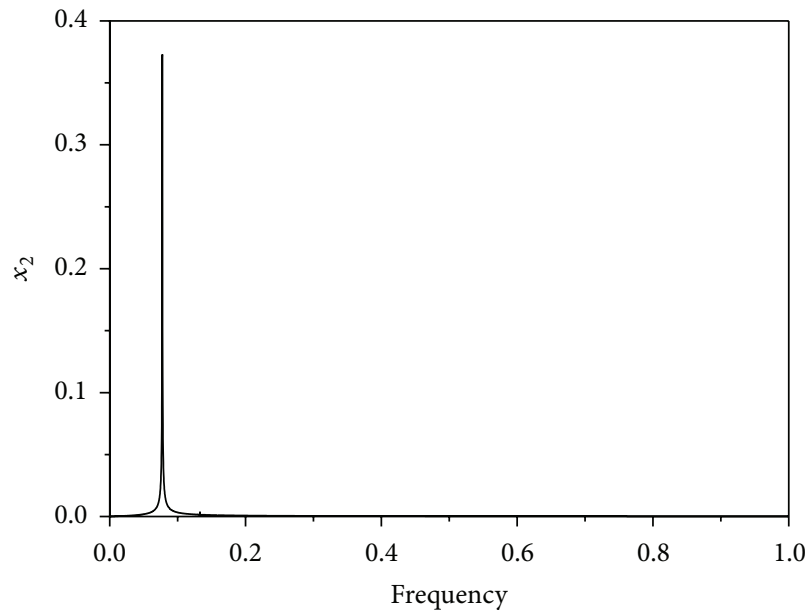

(b)

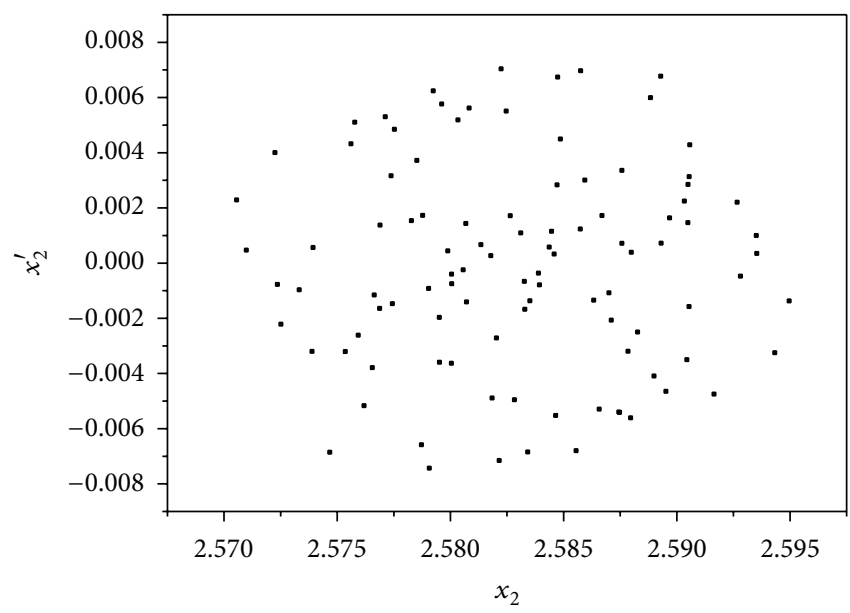

(d)

FIgure 14: See $\omega=1.30$. (a) Displacement response. (b) Frequency spectrum. (c) Phase-portrait plots. (d) Poincaré maps.

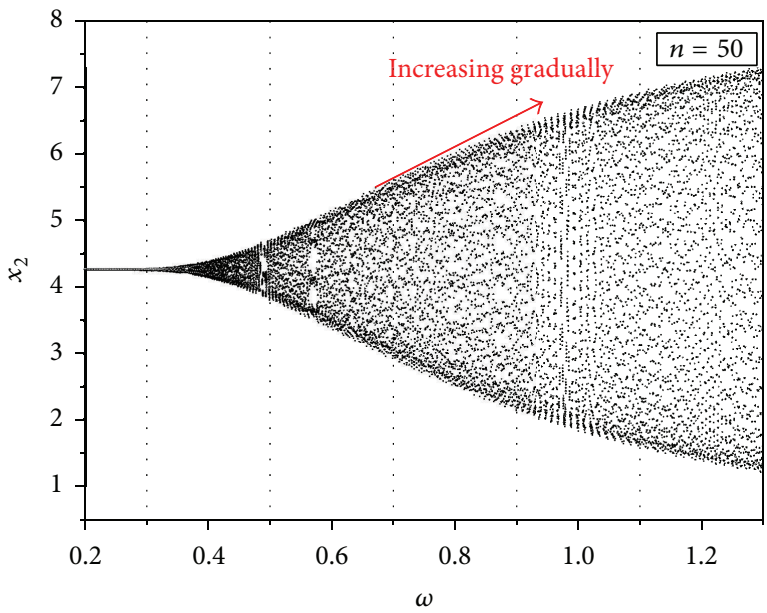

(a)

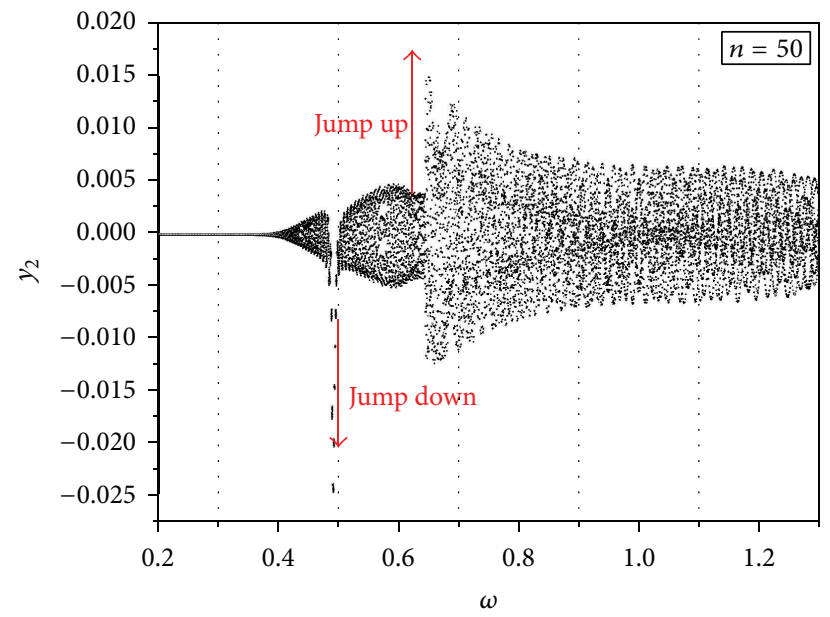

(b)

FIGURE 15: Steady-state response of displacement when $n=50$ : (a) $x_{2}$ and (b) $y_{2}$. 


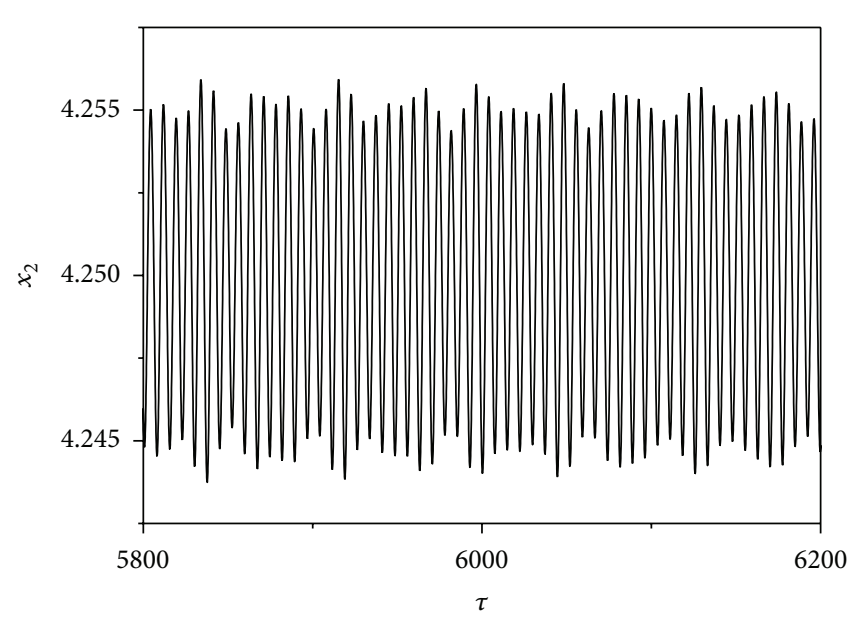

(a)

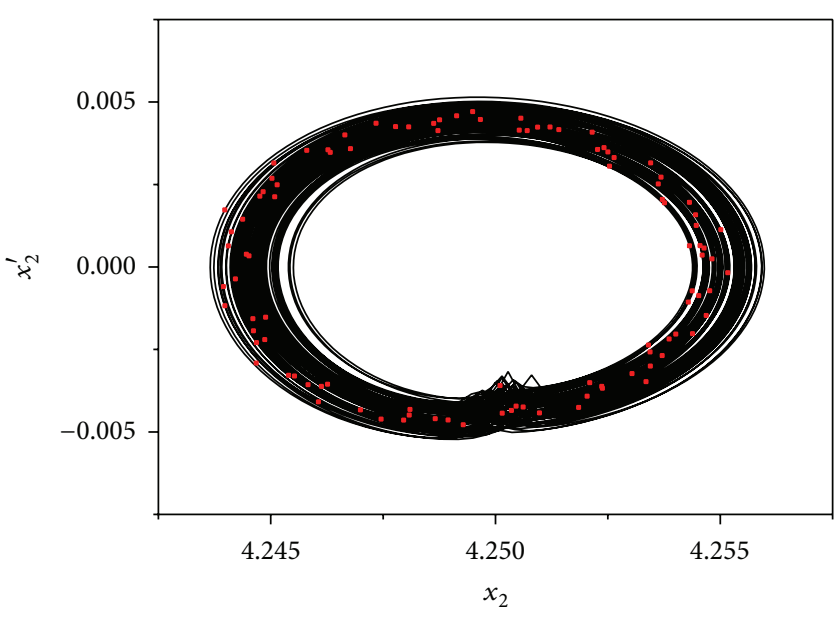

(c)

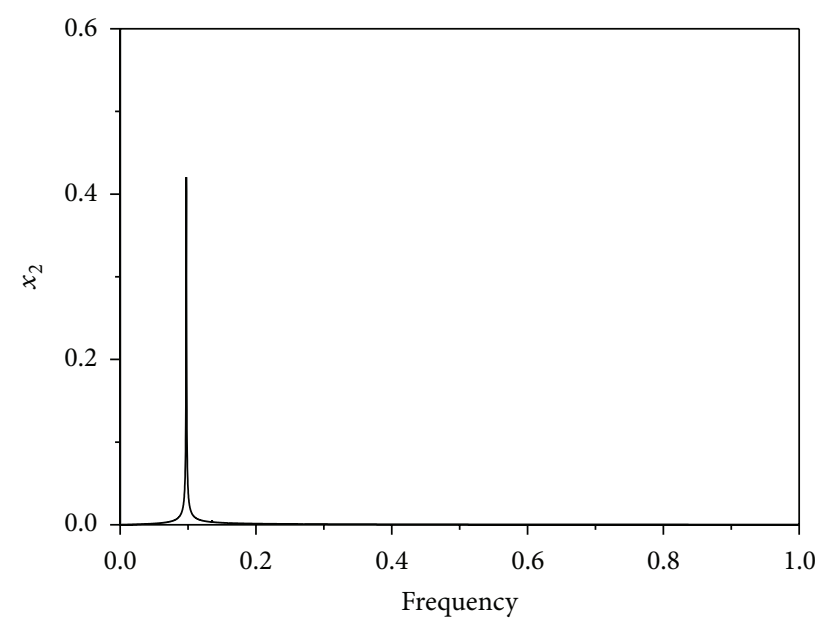

(b)

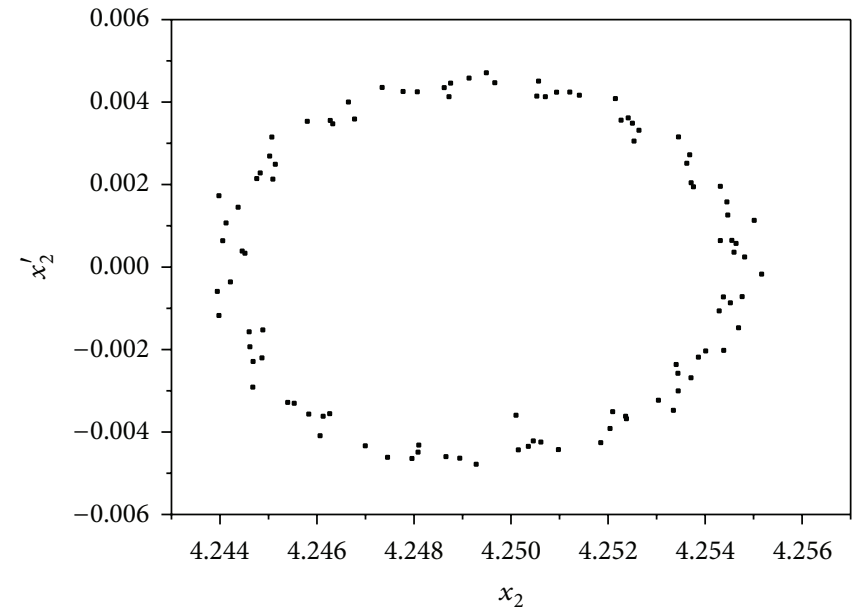

(d)

FIgURE 16: See $\omega=0.80$. (a) Displacement response. (b) Frequency spectrum. (c) Phase-portrait plots. (d) Poincaré maps.

$\omega=1.20$. The steady-state response of this system is confined to an annular region and makes a complex movement from the phase-portrait diagram. Besides, the distribution of the points on the Poincaré diagram demonstrates the chaotic motion. Thus, the motion is in the state of a chaos motion at this rotor speed.

Figure 18 illustrates the bifurcation diagram of floatingraft forced with an unbalanced excitation for the displacement responses $x_{2}$ and $y_{2}$ with respect to the motion parameter $\omega$, in which $\omega=0.20 \rightarrow 1.30$ in certain parameters for $k=12$. Increasing the rotor speed, the dynamic behaviors in $x_{2}$ and $y_{2}$ directions can be considered as single-one motion, quasi-motion, and chaos motion. With the increase of rotor speed, the amplitudes of air bag and floating-raft increase gradually. Moreover, the diagram shows sudden increment of $x_{2}$ and $y_{2}$ at $\omega=0.735$. The result indicates the relatively small reduction in displacements $x_{2}$ and $y_{2}$ of floating-raft with the increasing rotor speed, the specific situation in different case as shown in the flowing section.

Figure 19 shows the dynamic behavior of the system at $\omega=0.735$. The Poincaré diagram shows a nearly close curve distribution of the points. The steady-state response of this system is confined to an annular region and the phaseportrait plots show the system around circular orbit. Thus, the simulation results shown in Figure 19 confirm that at $\omega=$ 0.735 the motion can be considered quasi-periodic.

Diagrams of Figure 20 summarize the results of the numerical simulation at $\omega=1.10$. Phase-portrait plots are messy and frequency spectrum structure clearly shows that there exists a zero spectrum. The corresponding distribution of points in the Poincaré maps confirms the chaotic motion at $\omega=1.10$, and therefore we may conclude that chaotic motion occurring at these rotating speed ratio $\omega=1.10$.

Figure 21 plots the bifurcation diagram of the floatingraft with an unbalanced excitation. (a) and (b) represent the displacement responses $x_{2}$ and $y_{2}$ with respect to the motion parameter $\omega$, in which $\omega$ varied from 0.20 to 1.30 in certain parameters for $k=16$. The bifurcation diagram shows how the dynamic behaviors in $x_{2}$ and $y_{2}$ directions change from single-one motion to quasi-motion to chaotic motion, increasing the rotor speed. With the increase of rotor speed, the amplitude of air-floating increases gradually. 


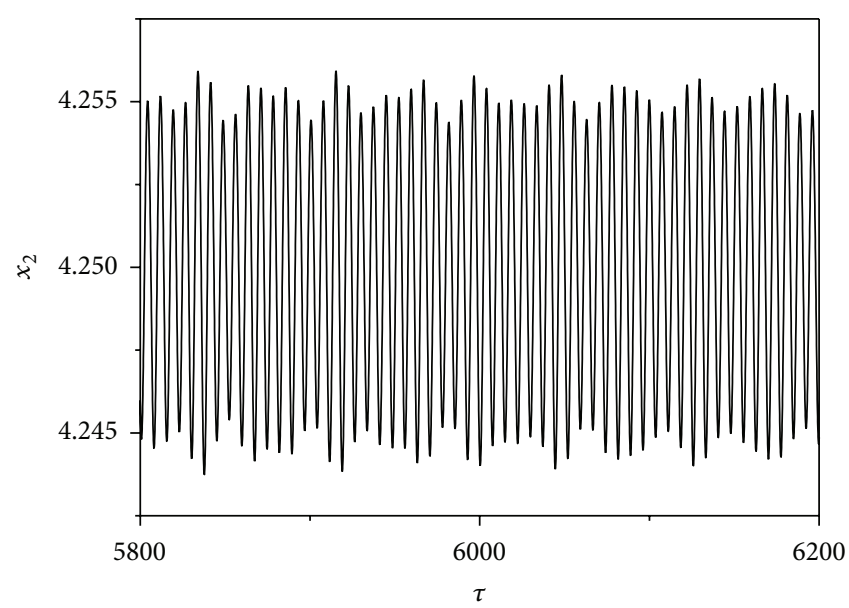

(a)

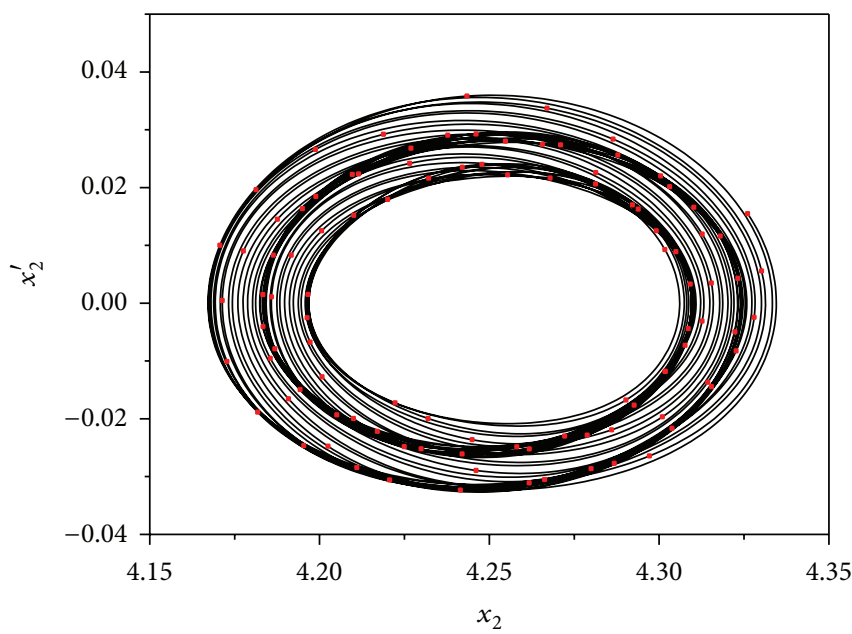

(c)

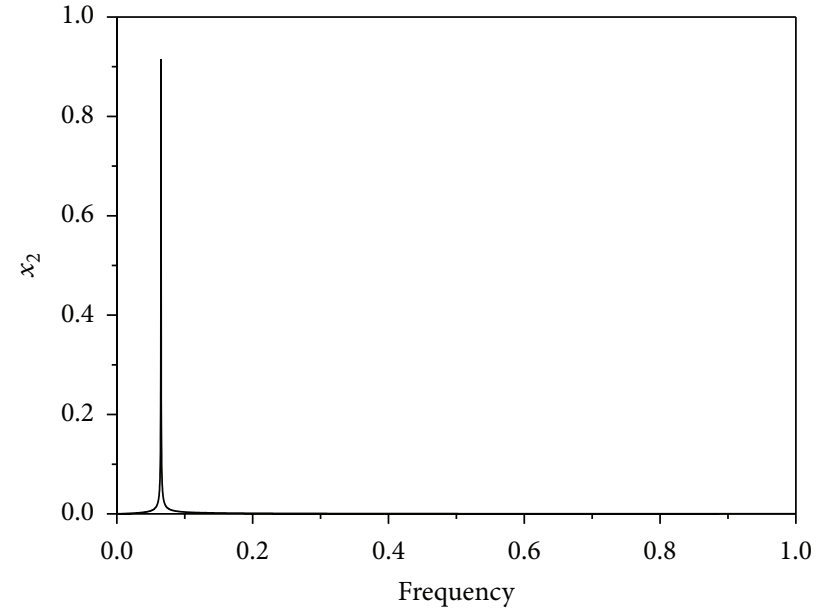

(b)

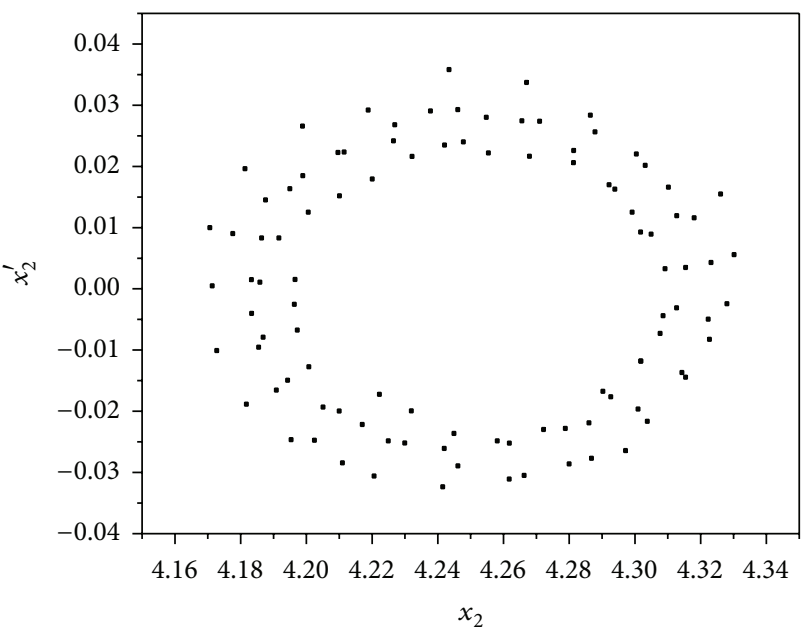

(d)

Figure 17: See $\omega=1.20$. (a) Displacement response. (b) Frequency spectrum. (c) Phase-portrait plots. (d) Poincaré maps.

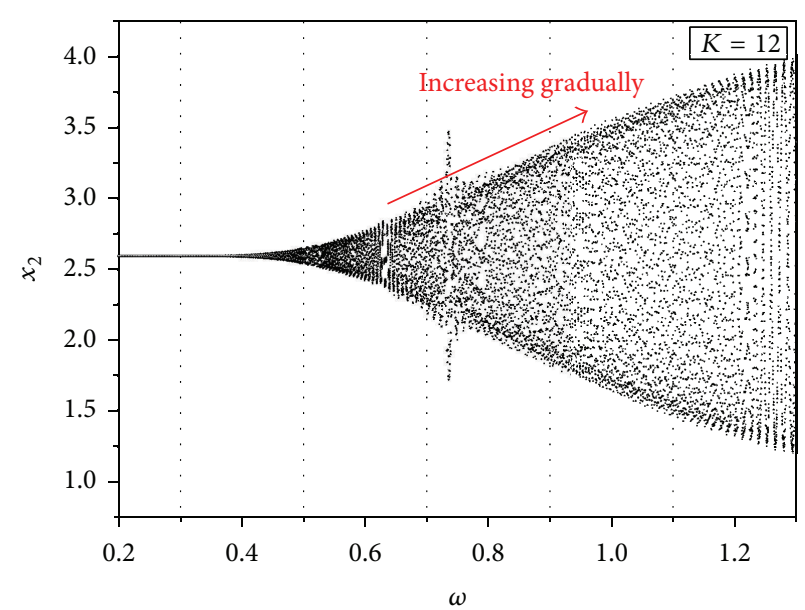

(a)

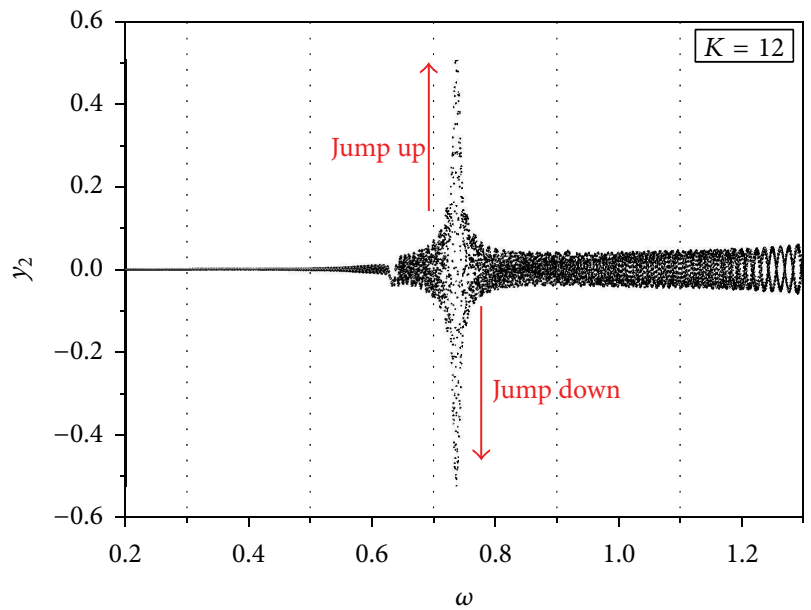

(b)

Figure 18: The steady-state response of displacement when $n=30$ : (a) $x_{2}$ and (b) $y_{2}$. 


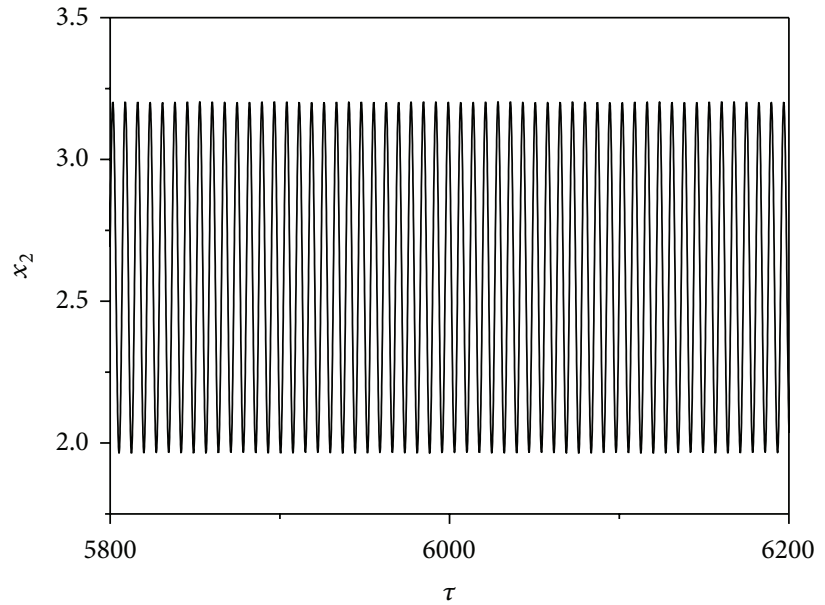

(a)

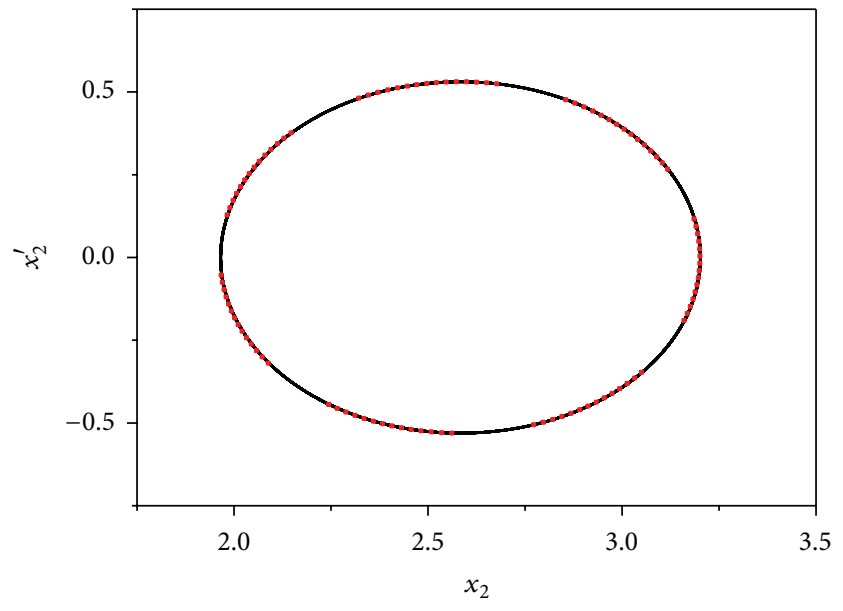

(c)

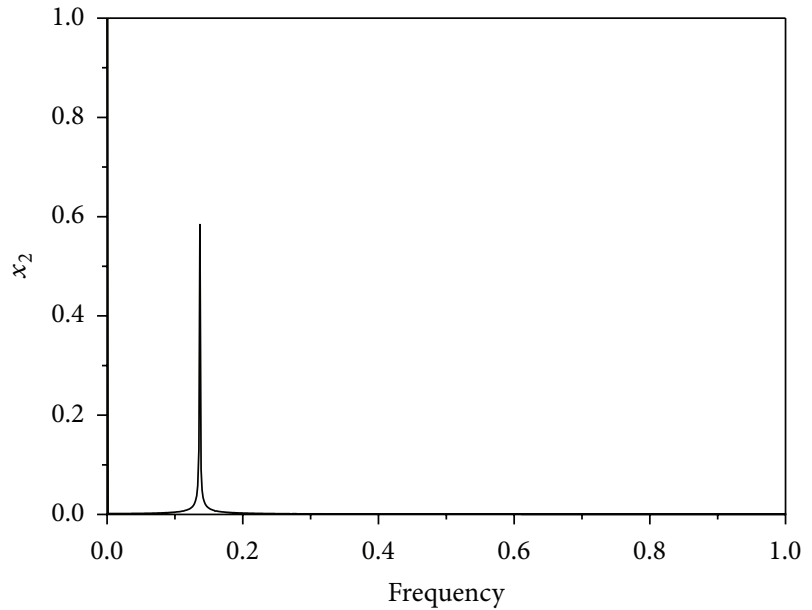

(b)

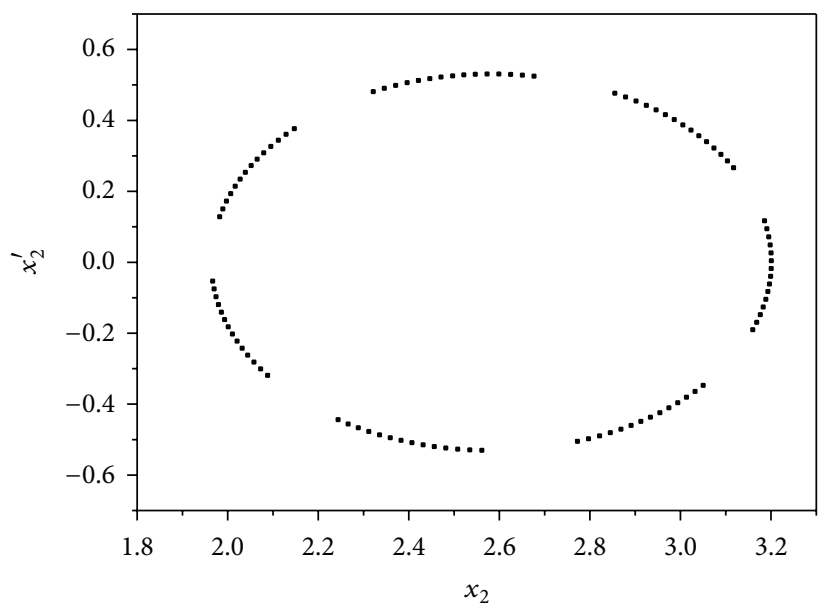

(d)

Figure 19: See $\omega=0.735$. (a) Displacement response. (b) Frequency spectrum. (c) Phase-portrait plots. (d) Poincaré maps.

At $\omega=0.835$, one observes a sudden increment of both $x_{2}$ and $y_{2}$ values. The result indicates the relatively small reduction in displacements $x_{2}$ and $y_{2}$ of floating-raft with increasing rotor speed, the specific situation in different case as shown in the flowing.

Figure 22 describes the dynamic response and its frequency spectrum, phase-portrait plots, and Poincaré map at $\omega=0.650$. The distribution of points of the Poincaré maps follows a closed curved; similarly, the steady-state response of the system is confined to an annular region and the orbit diagram shows that it moves regularly. Thus, at this rotor speed, the system can be considered in a quasi-periodic motion.

Figure 23 illustrates the chaotic motion of the nonlinear dynamics at $\omega=1.15$. Phase-portrait plots are messy, frequency spectrum structure clearly shows that there exists zero spectrum, and corresponding points on Poincaré maps are messy with irregular distribution. Therefore, the analysis of the simulation results confirms the chaotic motion at $\omega=$ 1.15 .

\section{Conclusions}

The nonlinear dynamical behavior of a rotor-bearing system coupled with air bag and a floating-raft support is carried out. In this paper, the mathematical model of the 4-DOFs system is developed according to the long bearing theory, and its nonlinear dynamic characteristics are studied by applying the Runge-Kutta method to solve motion equations. The result of this research greatly extends and enriches the understanding of the behavior of the system. Based on the results of the numerical simulations, the following main conclusions can be summarized.

(1) The responses at low speeds show a synchronous single periodic motion, and the unbalanced force plays a dominant role in the vibration of the rotorbearing system. With the increase of rotor speed, the influence of nonlinear oil force has great effect on the system vibration, and quasi-periodic motion can be generated. Meanwhile, there exists a quasi-periodic 


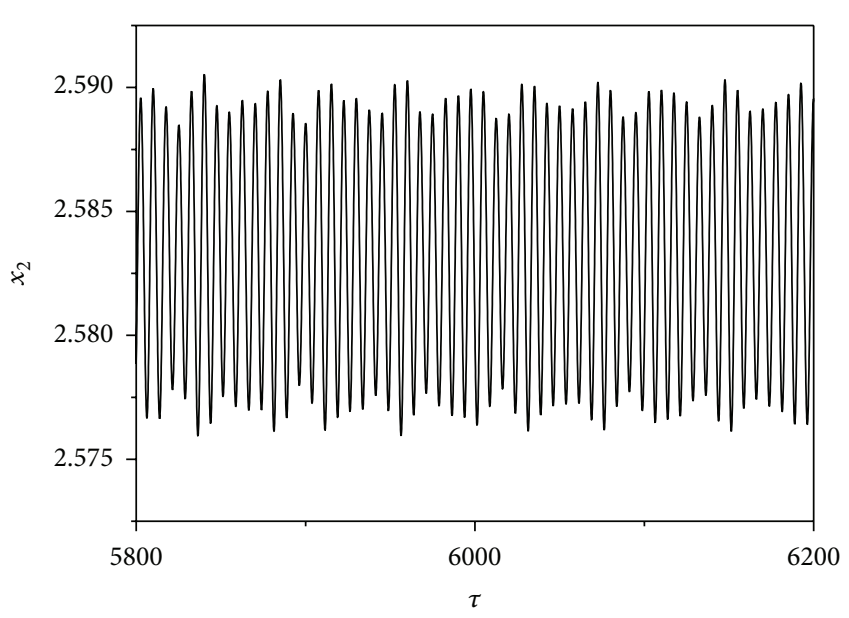

(a)

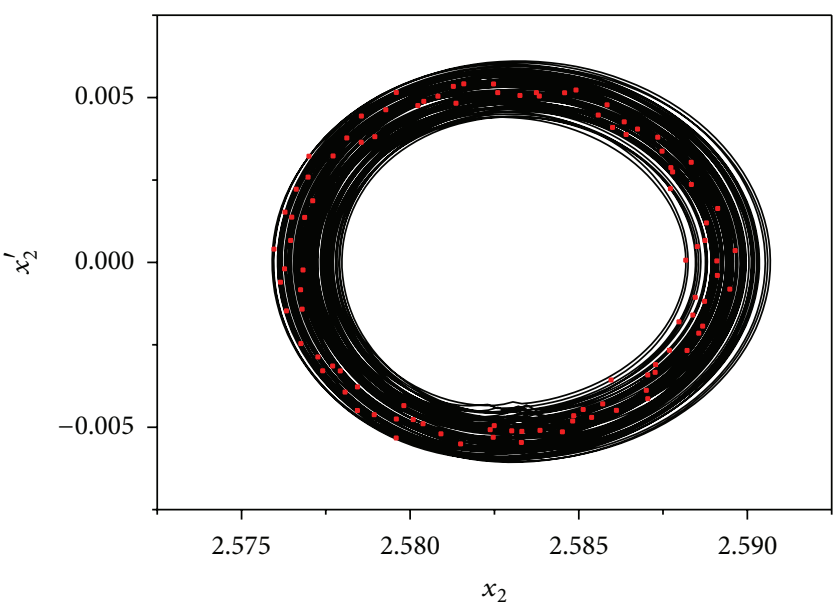

(c)

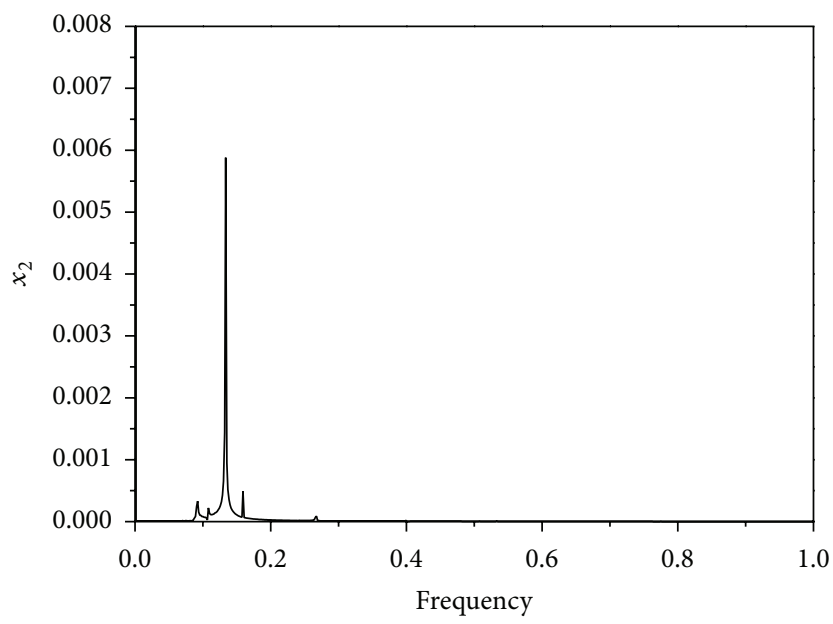

(b)

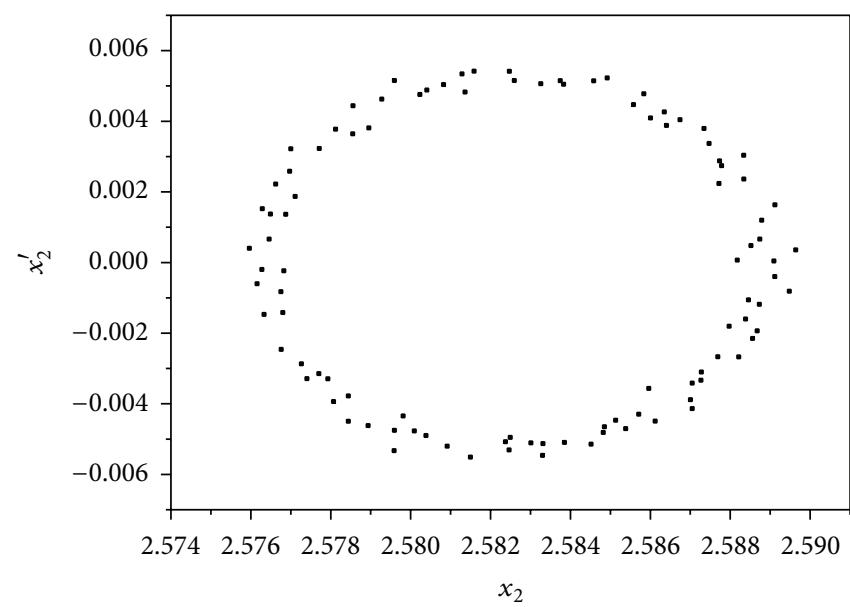

(d)

Figure 20: See $\omega=1.10$. (a) Displacement response. (b) Frequency spectrum. (c) Phase-portrait plots. (d) Poincaré maps.

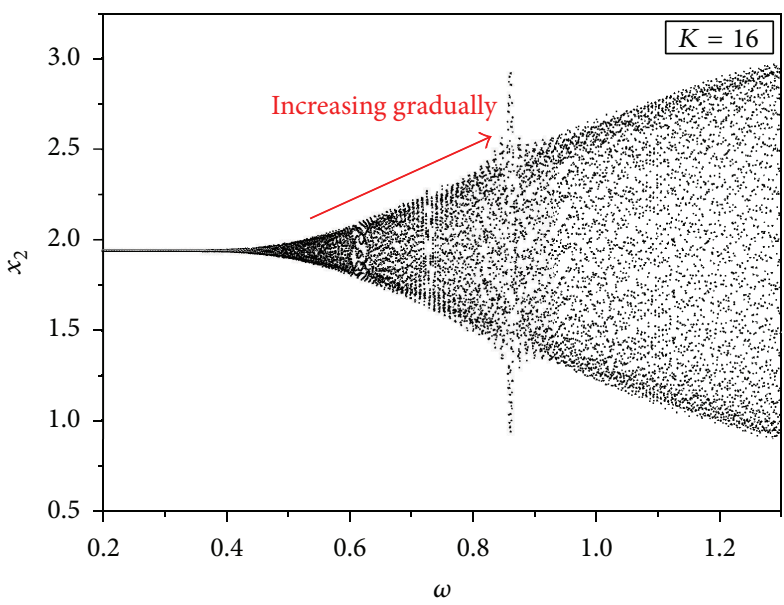

(a)

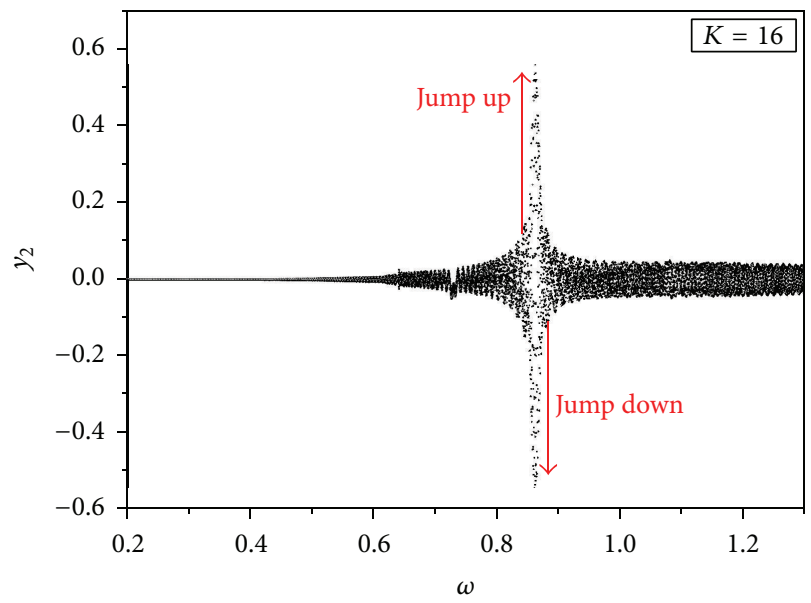

(b)

Figure 21: See $K=16$. (a) Displacement $x_{2}$ steady-state response. (b) Displacement $y_{2}$ steady-state response. 


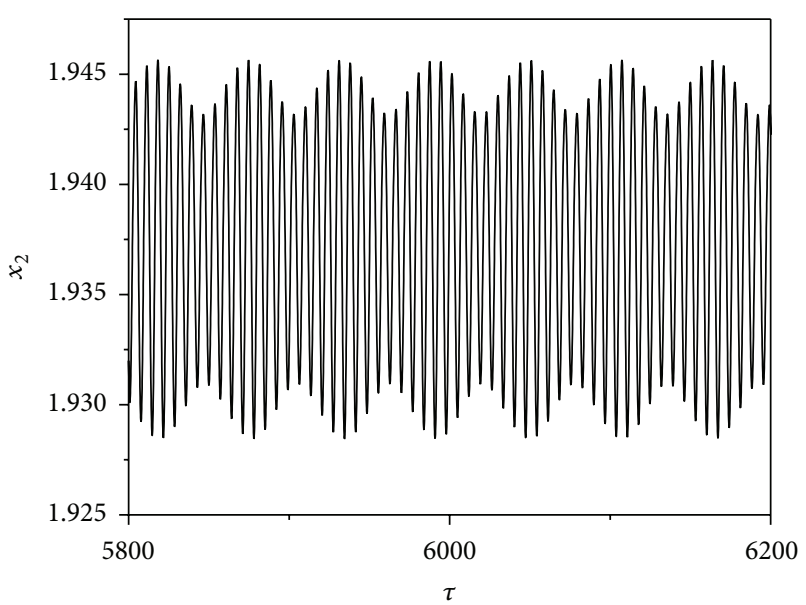

(a)

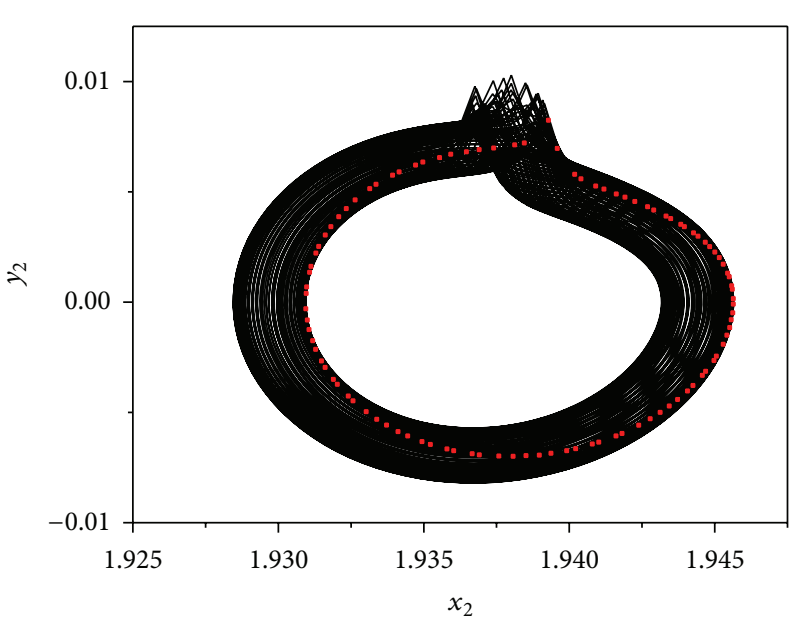

(c)

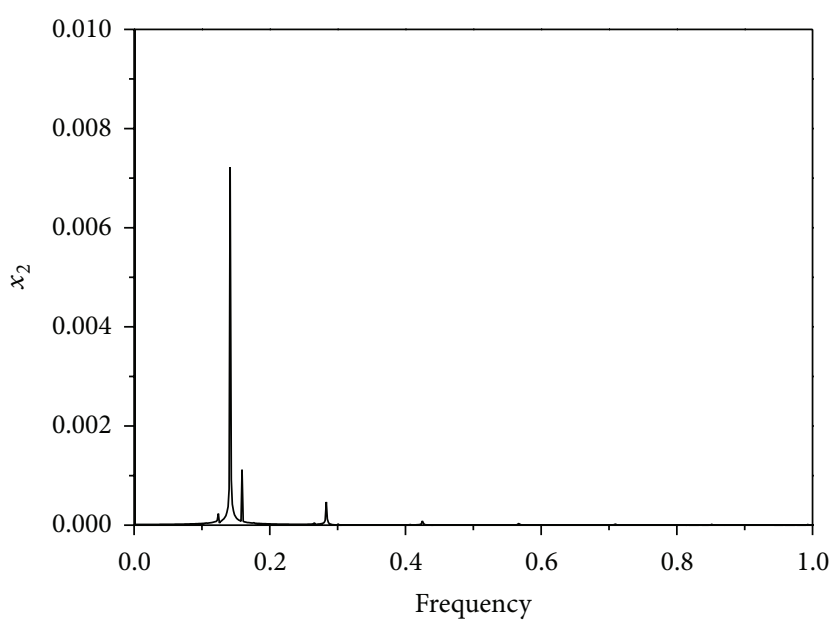

(b)

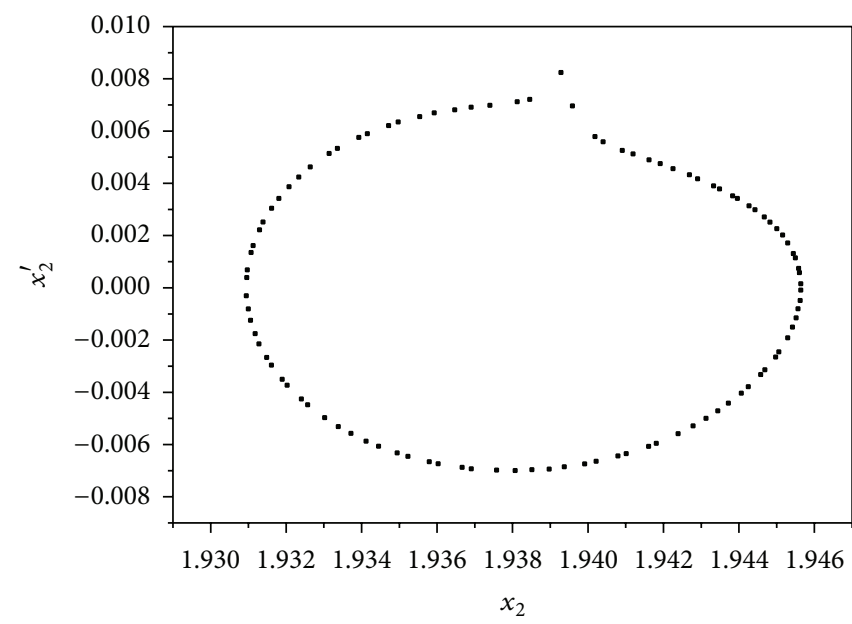

(d)

Figure 22: See $\omega=0.650$. (a) Displacement response. (b) Frequency spectrum. (c) Phase-portrait plots. (d) Poincaré maps.

bifurcation phenomenon. As a result, the system at high rotor speeds gradually moves into the complex and irregular state of chaotic motion.

(2) The dynamic properties of floating-raft at different key system parameters along with ratio speed generate important nonperiodic motion. The result of numerical modeling of the system suggests that some designing parameters change the dynamic stability. Moreover, periodic motions, quasi-periodic motion, and chaotic motion can appear and enhance the understanding of the nonlinear dynamics of floatingraft and may help in the theoretical understanding of general nonlinear systems to help design ideal isolation equipment.

These dynamic properties can be used to provide the theoretical guidance of parameter design of a rotor-bearing system coupled with air bag and floating-raft.

\section{Appendix}

\section{Notations}

c: $\quad$ Bearing clearance

$e: \quad$ Eccentricity position of rotor center-line in journal bearing

$d$ : Dimensionless damping

$D$ : Damping damping

$f_{x}, f_{y}$ : Dimensionless oil film forces of journal bearing

$F_{x}, F_{y}$ : Oil film forces of journal bearing

$g$ : The acceleration of gravity

$h$ : Thickness of oil film

$k$ : $\quad$ Dimensionless stiffness

$K: \quad$ Dimensional stiffness

$m_{1}$ : The mass of disc

$m_{2}$ : The mass of floating-raft

$n: \quad$ The ration of mass

$p$ : Oil film pressure 


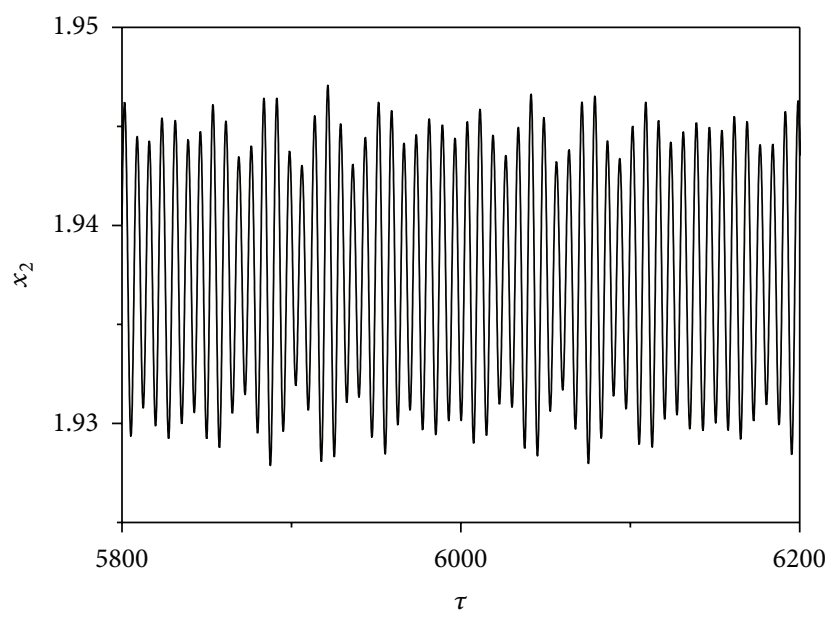

(a)

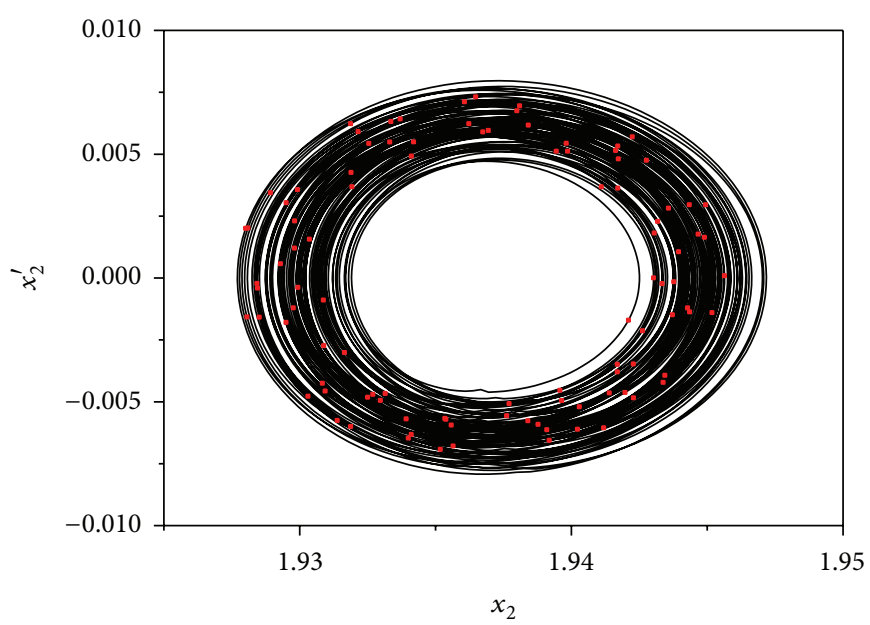

(c)

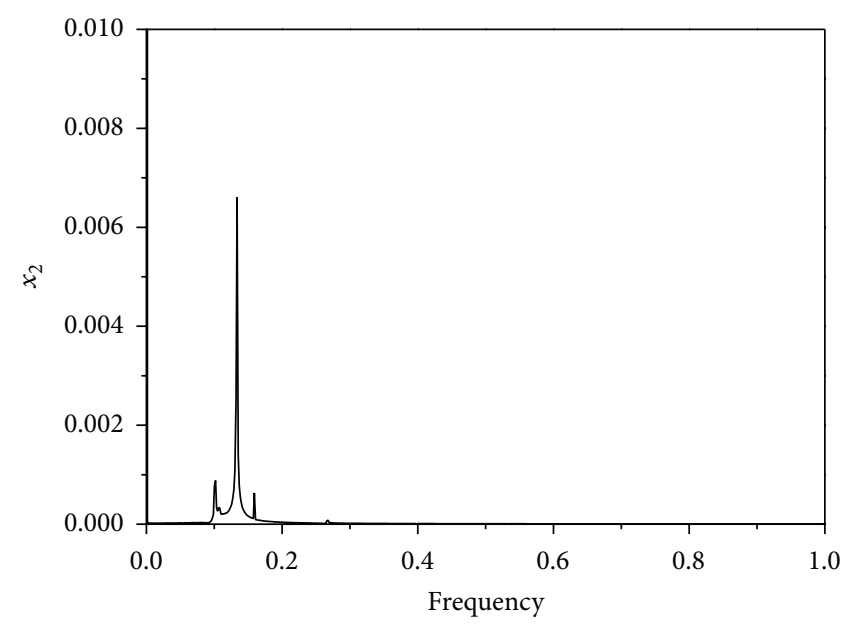

(b)

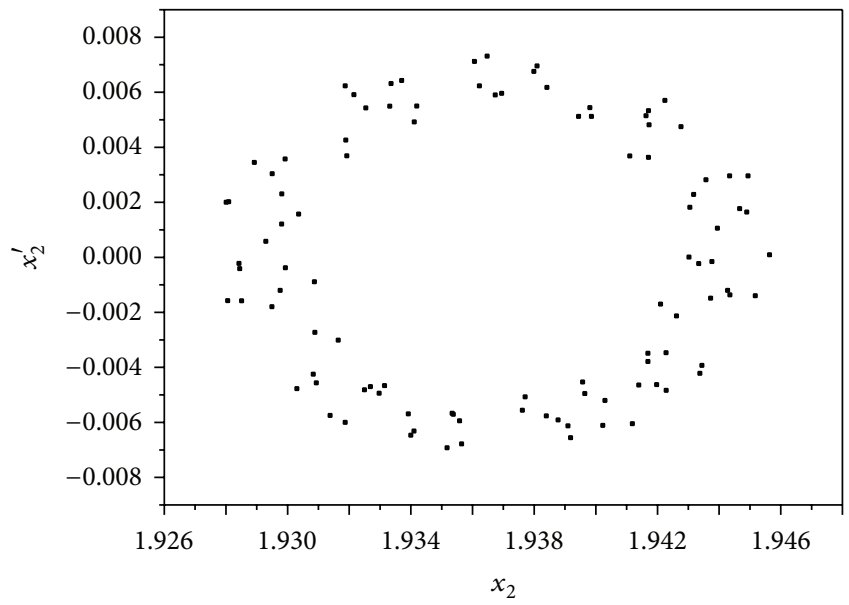

(d)

Figure 23: See $\omega=1.15$. (a) Displacement response. (b) Frequency spectrum. (c) Phase-portrait plots. (d) Poincaré maps.
t: $\quad$ Time
u: Vector variable
$x, y$ : Coordinates of the centers of disk
$z, \theta$ : Coordinates in width and circumference
$\alpha$ : Directions of dimensionless eccentricity
$\varepsilon$ : Bearing eccentricity
$\varphi$ : Angular position of rotor center-line in journal bearing
$\sigma: \quad$ Sommerfeld of bearing
$\lambda: \quad$ The ratio of length diameter
$\tau$ : Dimensionless time
$\Omega: \quad$ Rotating speed of rotor
$\omega$ : Dimensionless rotor speed.

\section{Subscripts}

1, 2: Disc (or rotor-bearing) and floating-raft.
Superscripts

., ..: First- and second-order derivatives with time $t$

I, II: First-order and second-order derivatives with dimensionless time $\tau$

$\tau: \quad$ Nondimensional time $\tau$.

\section{Conflict of Interests}

The authors declare that there is no conflict of interests regarding the publication of this paper.

\section{Acknowledgments}

This work was supported by the National Natural Science Foundation of China (Grant no. 11372245) and the Natural Science Foundation of Shaanxi Province, China (Grant no. 2014JM1015). This support is gratefully acknowledged. 


\section{References}

[1] Z. G. Wang, "Dynamic modeling and response analysis to impact of elastic floating raft system," Journal of Ship Mechanics, vol. 9, no. 6, pp. 113-125, 2005.

[2] J. C. Niu, K. J. Song, and G. Zhao, "Research on vibration of an active plate-like floating raft system," Chinese Journal of Mechanical Engineering, vol. 40, no. 5, pp. 67-71, 2004.

[3] X. Zhang, Q. W. He, and X. Yu, "Modeling and dynamic analysis for rigid-flexible coupling nonlinear floating raft vibration isolation system," Applied Mechanics and Materials, vol. 34-35, pp. 1994-1998, 2010.

[4] J.-C. Niu and K.-J. Song, "Active control strategies of a floating raft vibration isolation system for marine diesel engines," Transactions of CSICE, vol. 22, no. 3, pp. 252-256, 2004.

[5] Z.-G. Wang, Q. Feng, and Y. Wang, "Dynamic modeling and response analysis to impact of elastic floating raft system," Journal of Ship Mechanics, vol. 9, no. 6, pp. 113-125, 2005.

[6] G. H. Jiang, L. G. Yin, R. Y. Shen, and G. M. Wu, "Experimental study on non-linear shock response of shipboard equipment in consideration of elasticity of raft body and base," Journal of Vibration and Shock, vol. 25, no. 2, pp. 109-112, 2006.

[7] Q. B. Huang, "Finite element analysis of floating raft isolation system based on complex stiffness method," Hua Zhong Ke Ji Daxue Xuebao, vol. 35, no. 1, pp. 99-101, 2007.

[8] M. Stephen, "Analytical modeling of single and two-stage vibration isolation system," in Proceedings of the Australian Acoustical Society Conference, pp. 337-344, Gold Coast, Australia, November 2011.

[9] F. Wang, C. Wang, L. Guo, B. Wen, and Y. Li, "Study on floating raft vibration isolation system for pumping unit," Advanced Materials Research, vol. 383-390, pp. 130-135, 2012.

[10] J. C. Niu and K. J. Song, "Research on vibration isolation of an active plate-like floating raft system," Journal of Mechanical Engineering, vol. 40, no. 5, pp. 67-71, 2004.

[11] C.-W. Chang-Jian, "Non-linear dynamic analysis of dual flexible rotors supported by long journal bearings," Mechanism and Machine Theory, vol. 45, no. 6, pp. 844-866, 2010.

[12] G. Adiletta, A. R. Guido, and C. Rossi, "Chaotic motions of a rigid rotor in short journal bearings," Nonlinear Dynamics, vol. 10, no. 3, pp. 251-269, 1996.

[13] X. H. Wu, R. Q. Liu, and S. B. Xia, "The whirl orbit and stability of journal bearing under the nonlinear oil film force," Journal of Vibration Engineering, vol. 9, no. 3, pp. 302-307, 1996.

[14] W. Kim, H. H. Yoo, and J. Chung, "Dynamic analysis for a pair of spur gears with translational motion due to bearing deformation," Journal of Sound and Vibration, vol. 329, no. 21, pp. 4409-4421, 2010.

[15] X. F. Li, L. X. Zheng, and Z. Liu, "Balancing of flexible rotors without trial weights based on finite element modal analysis," Journal of Vibration and Control, vol. 19, no. 3, pp. 461-470, 2013.

[16] W. Zhao and M. Li, "Study of rotor-bearing system coupled with a floating raft isolation device," Applied Mechanics and Material, vol. 598, pp. 202-205, 2014.

[17] P. Song, X.-J. Dong, and G. Meng, "Dynamic reduction modeling and time delay for an active vibration isolation system with flexible base," Journal of Vibration and Shock, vol. 31, no. 23, pp. 57-61, 2012. 

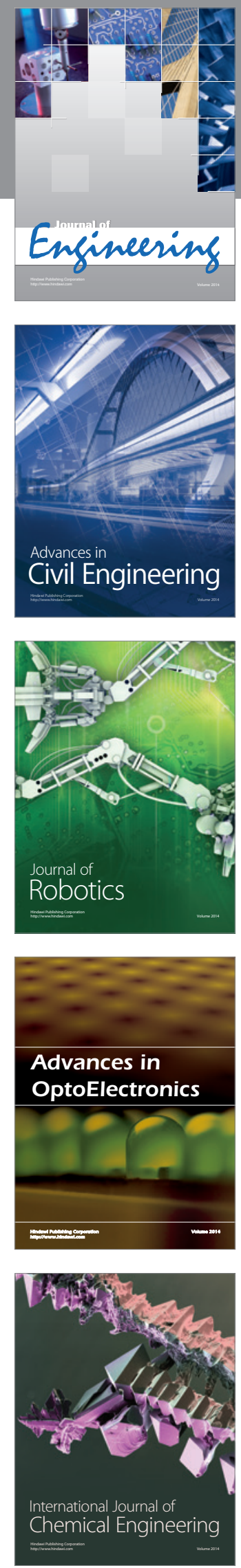

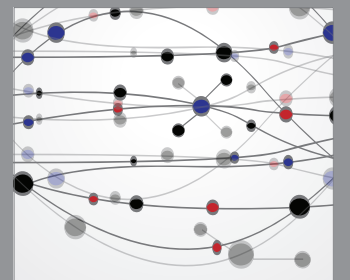

The Scientific World Journal
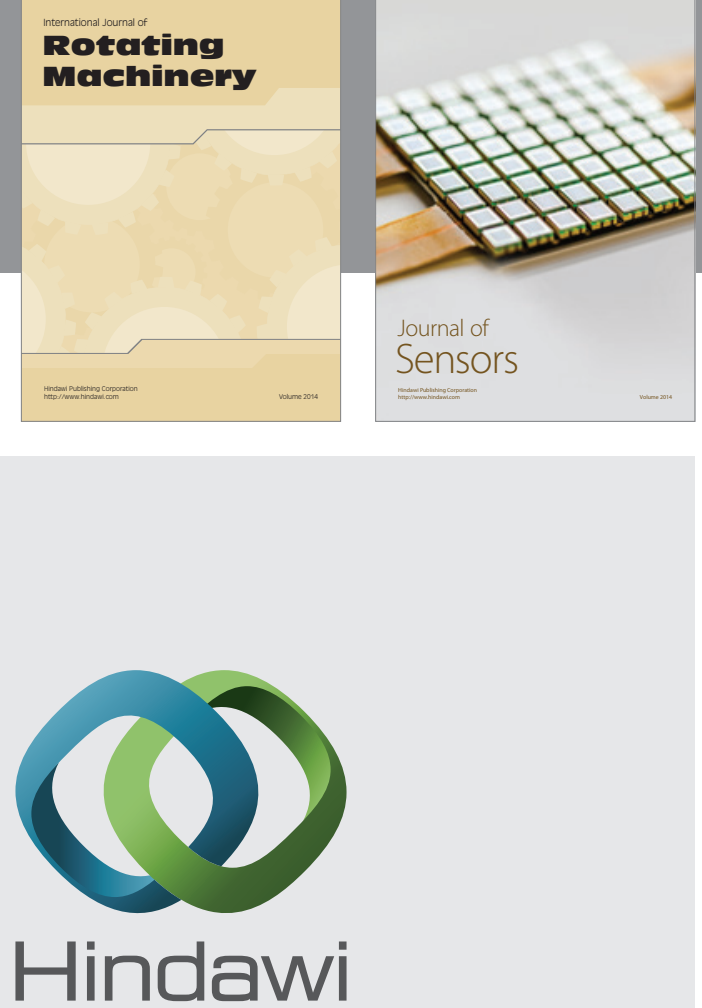

Submit your manuscripts at http://www.hindawi.com
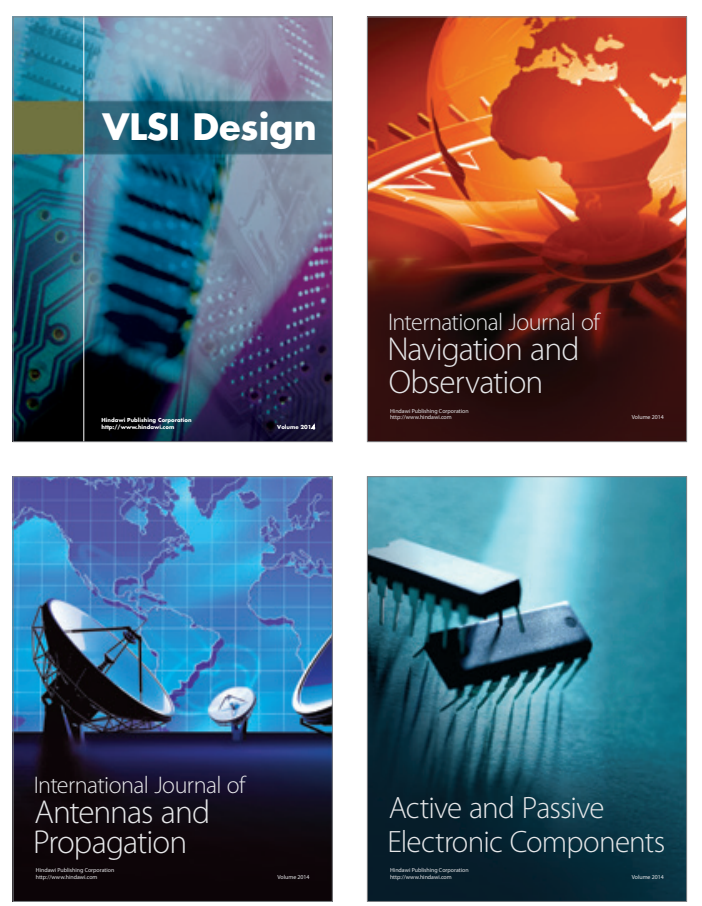
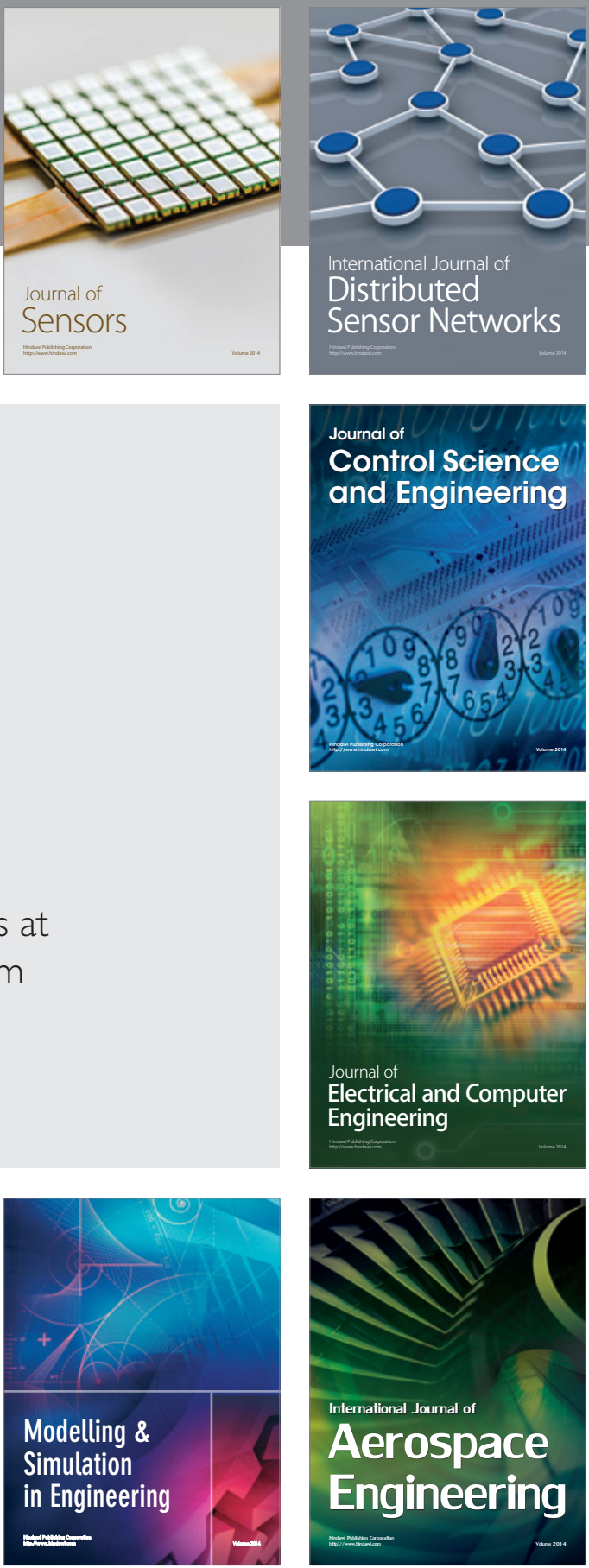

Journal of

Control Science

and Engineering
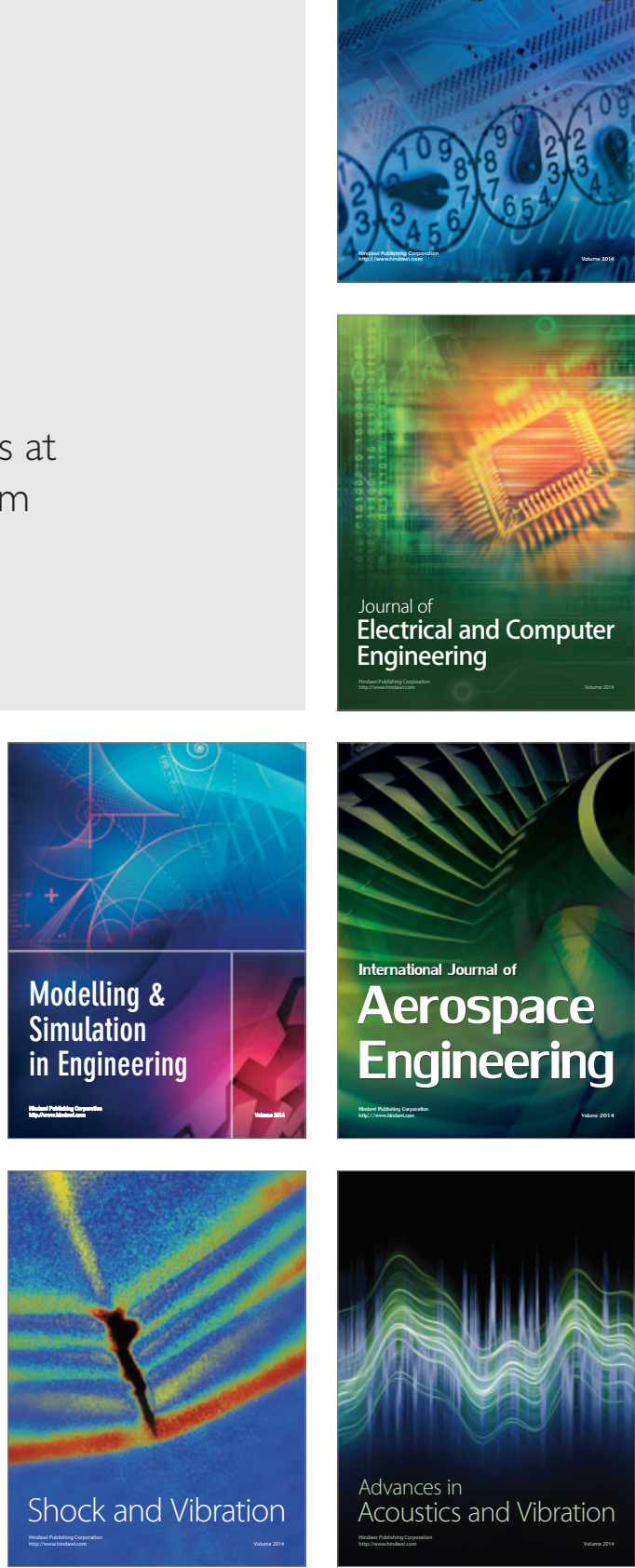